\title{
Efficiency of Semi-Autonomous and Fully Autonomous Bus Services in Trunk-and-Branches Networks
}

\author{
Wei Zhang $\mathbb{D}$, Erik Jenelius $\mathbb{D}$, and Hugo Badia $\mathbb{1}$ \\ Division of Transport Planning, KTH Royal Institute of Technology, SE-100 44 Stockholm, Sweden \\ Correspondence should be addressed to Wei Zhang; wzh@kth.se
}

Received 14 October 2018; Revised 25 January 2019; Accepted 5 February 2019; Published 27 February 2019

Academic Editor: Ludovic Leclercq

Copyright (C) 2019 Wei Zhang et al. This is an open access article distributed under the Creative Commons Attribution License, which permits unrestricted use, distribution, and reproduction in any medium, provided the original work is properly cited.

\begin{abstract}
Automation technology is expected to change the public transport sector radically in the future. One rising issue is whether to embrace the intermediate stage of semi-autonomous buses or to wait until fully autonomous buses are available. This paper proposes a cost model of bus operations considering automation technology. The generalized cost, which is the sum of waiting, riding, operating, and capital cost, is modeled for conventional, semi-autonomous, and fully autonomous bus services on a generic trunkand-branches network. Semi-autonomous buses achieve reduced unit operating cost through automated platooning on the corridor. The relative efficiency of the different services is studied under a range of scenarios for commercial speed, network structure, and demand distribution. Analytical and numerical results show that fully autonomous buses exhibit great potential through reduced operating and waiting costs even if the additional capital cost is high. The advantages of semi-autonomous buses are weaker and most prominent in networks with low demand along a long corridor such as interurban networks. For both automation levels a commercial speed comparable to conventional vehicles is crucial. The established criteria provide input to planners and operators for understanding the potential of automated bus services.
\end{abstract}

\section{Introduction}

Autonomous vehicles (AV) have progressed through conceptual design, fundamental research, and technological development and are now facing commercial applications. Automation technology has the potential to significantly change and in many ways improve current mobility, as summarized by Fagnant and Kockelman [1]. Autonomous vehicles may dramatically reduce the number of crashes due to distraction, inexperience, intoxication, etc. and enable smoother speed adjustments and shorter intervehicle distances compared to conventional vehicles, which could result in lower fuel consumption and higher lane capacity. Traditional car ownership may transition towards a shared mobility, where vehicles pick up travelers at the origins and send them to the desired destinations. Given an adequate penetration rate, fewer private cars will be required and the need for parking space may also be reduced. However, the vehicle miles traveled (VMT) will increase due to unoccupied relocation of the SAV [2].
Bösch et al. [3] conduct a comprehensive evaluation of fully autonomous vehicles for different operation modes, including line-based transit, taxi, and private car. The authors conclude that fleets of SAV will be slightly more cost-effective than other modes. Furthermore, emerging transport modes will have a great impact on the conventional mass transit system and competition may appear. In terms of the bus transit system, the authors predict that fully autonomous buses with smaller capacities and higher frequencies may be a future direction, due to the labor savings. Autonomous vehicles are also believed to be an efficient way to solve the first-/last-mile problem of conventional public transport. For example, Scheltes and Correia [4] use an agentbased simulation model to estimate the performance of an Automated Last-Mile Transport (ALMT) system where a fleet of fully autonomous electric vehicles is used to serve the passengers traveling between the train station and the university campus. The result shows that the ALMT can potentially reduce waiting time and travel time compared to walking and cycling. However, congestion may appear 
if the interaction between vehicles and surroundings is considered.

While there is a growing research literature around automated shared mobility as a competitor or supplement to existing public transport, research about automation in the bus transit system itself is limited. Bergqvist and Åstrand [5] establish a linear programming model to investigate how autonomous minibuses (15-passenger capacity) will impact four high-frequency bus lines in Stockholm, Sweden. Using the numbers of conventional buses and minibuses as decision variables, the hourly total operating cost is minimized. The results show that introducing autonomous buses into the current bus transit system can significantly reduce the operating cost. Due to data unavailability, the study did not consider other cost components. For the purpose of real-world implementation, Lam [6] develops a simulation platform to efficiently manage the autonomous public transportation system by using dynamic origin-destination matrix, including bus scheduling, cooperative maneuvering, and priority management at road intersection. The platform is shown to be viable to determine the optimal fleet size, vehicle capacity, and service frequency.

The introduction of new technologies in the public transport system usually involves a series of updates in infrastructure, land use, and human resources distribution, which makes related analysis different from other transport modes. Besides, the level of service is subject to customers' satisfaction and should also be compatible with the updated system. The advantages of new technologies should be evaluated not only by the first-stage investment from the service provider but also by the subsequent operating scheme which affects both service provider and customers. Therefore, the optimization of the operating scheme in public transport is indispensable for promising technologies like automation to improve social welfare to the greatest extent. In addition, it is also crucial for the service provider and policy makers to decide when to enter the emerging market, since automation technology keeps evolving and the capital cost may vary from time to time.

The objective of this study is to formulate a cost model for comparing bus services that differ with respect to the level of automation. To the best of our knowledge, bus service modeling considering different automation levels, especially involving semi-autonomous buses, has not been previously addressed in the literature. Moreover, the identified scenarios of where and which type of autonomous buses can be more favorable than conventional buses contribute to the existing relevant research which mostly regards autonomous vehicles as a supplement of the traditional public transport system. The motivation behind this study is to shed light on the impact and importance of different parameters and highlight those which should be included in further analysis.

Analytical cost models are widely used for the study of transport systems and in particular for the comparison of different aspects of transit systems, such as type of services [8] or network structures [9]. It is assumed that different levels of automation will result in different labor costs (higher levels of automation require less human driver participation) and capital costs (automation requires more expensive technology) as well as variations in bus operating modes associated with (or restricted by) each bus type. The paper considers the minimization of the generalized cost (waiting cost, riding cost, operating cost, and capital cost) using bus size as the decision variable; no subsidies from the government are considered. However, this study does not fall into the category of the traditional bus size or bus service optimization problem, although it encapsulates the common process of bus service optimization. The realization of bus size affects other service characteristics, such as frequency and fleet size. The service characteristics together determine each cost component, among which trade-offs may occur. Both analytical solutions and numerical studies are provided, for the purpose of general use and sensitivity analysis.

The paper considers two levels of bus service automation:

Semi-automation: Semi-autonomous buses represent a transition (or intermediate) state between conventional buses and fully autonomous buses. More precisely, if we regard the level of automation for conventional buses as 0 (no automation) and for fully autonomous buses as 5, the semiautonomous buses belong to level 4 (high automation). According to Daniel and Martin [10] some assistant functions (e.g., front collision warning and park distance control) that are already available in the market fall into the level 0 category, even though these modules may be perceived to involve automation technology. This means that some driving mode-specific actions do not require drivers to respond, because it is the system rather than the driver that monitors and reacts to the environment. Level 4 automation enables operating schemes involving cooperative bus platooning. By forming bus platoons, all buses experience labor saving except for the leading one, which requires a conventional human driver. Typically, all vehicles are equipped with $\mathrm{V} 2 \mathrm{~V}$ communication technology and radars to measure the distance to the vehicle ahead. The intervehicle distance in a platoon can be very short (and adjustable according to road conditions), but the buses are not attached. Compared with articulated buses, bus platoons are more flexible and safer in terms of steering and are less likely to block road junctions. Unlike double-decker buses, single-decker bus platoons require shorter dwell times and are less restricted by low bridges. Autonomous bus platooning in public transit is studied conceptually in Antonio Loro Consulting Inc. [11], which predicts the bus platooning technology should ideally be implemented within approximately 3 to 10 years. Several real-world test cases on bus platooning can be found in TRB's E-Circular 232 [12]. To form bus platoons, coordination is needed so that multiple buses can drive together on the common path [13-15]. Most studies on vehicle platooning assume that all vehicles have manual drivers, and the focus is on fuel savings (e.g., [13]). However, when the vehicle speed is low (e.g., less than $55 \mathrm{~km} /$ hour), which is true for buses, the air drag and consequently the fuel savings from platooning are typically negligible. Therefore, our study focuses on the labor cost savings rather than fuel savings.

Full automation: Under full automation (Level 5), all buses can operate without drivers, which reduces labor costs. Coordination is not needed but the capital cost of fully 
autonomous buses is expected to be higher than for semiautonomous buses.

The remainder of the paper is structured as follows. Section 2 introduces the modeling of different levels of automation in capital cost and operating cost. The network structure, generalized cost framework, and optimal service provision are described in Section 3. In Section 4, the comparison between autonomous buses and conventional buses is carried out with respect to the service characteristics, costs, and benefits for the service provider and the travelers. Numerical analysis of the impacts of parameters related to cost, network structure, and demand profile for the applicability of the different vehicle technologies is presented in Section 5. Section 6 concludes the paper.

\section{Bus Cost Structure}

This section presents the models for the operating and capital costs of conventional, semi-autonomous, and fully autonomous buses. The notation adopted in the paper is summarized in Table 1.

2.1. Conventional Bus Cost Structure. Regarding the service provider's cost, different terms and classifications appear in previous studies. In Jansson [16], bus company cost consists of traffic operating cost $(80 \%)$ and overhead cost (20\%). The former includes crew cost (50\%), bus capital cost (25\%), and other costs (fuel, repair, maintenance, insurance, tax, etc., 25\%), whereas the latter contains administrative cost, pensions, etc. The bus capital cost and other costs are divided into running cost and standing cost. Basically, the running cost is proportional to the driving distance, while the standing cost is proportional to the time of usage. Another basis of variation is peak vehicle, which mainly affects depreciation and leasing, interest on capital debt, etc. [17]; this factor is not considered here. Thus, the bus company cost is modeled as the sum of crew cost, standing cost, running cost, and overhead cost.

To avoid ambiguities in terminology, we use operating cost to refer to the sum of on-vehicle crew cost, fuel, tires, vehicle repair and maintenance, dispatching, and overhead. Meanwhile, the capital cost includes the capital cost of the vehicle, the cost of rehabilitation, and residual value at disposal [7]. The hourly operating cost can be divided into two categories: time-related operating cost and distance-related operating cost. Assuming that the vehicle operates at a fixed commercial speed (approximately $15 \mathrm{~km} /$ hour in urban areas), the distance-related operating cost can be easily converted to time-related operating cost.

To model the bus company cost with respect to the bus size, we refer to Jansson [16], who reports a linear relationship between the running cost and the bus size based on statistical analysis. This linear dependency also appears when the crew cost is added to the standing cost. The linear relationship is used in several subsequent studies [18-21]. The operating cost per vehicle hour is thus modeled as a linear function of vehicle size (seated and standing passenger capacity) $s^{c}$, with two
TABLE 1: Notation.

\begin{tabular}{|c|c|}
\hline Notation & Description \\
\hline$s$ & vehicle size (seated and standing capacity) \\
\hline$h$ & headway \\
\hline$n$ & fleet size \\
\hline$m$ & number of branches \\
\hline$C_{\text {tot }}$ & total cost \\
\hline$C_{\text {wait }}$ & waiting cost \\
\hline$C_{\text {ride }}$ & in-vehicle travel time cost \\
\hline$C_{\text {oper }}$ & operating cost \\
\hline$C_{\mathrm{cptl}}$ & capital cost \\
\hline$g_{\text {oper }}$ & operating cost per vehicle hour \\
\hline$g_{\text {cptl }}$ & capital cost per vehicle hour \\
\hline$c_{\text {ride }}$ & unit in-vehicle travel time cost \\
\hline$c_{\text {wait }}$ & unit waiting time cost \\
\hline$c_{\text {oper }}$ & unit fixed operating cost per vehicle-hour \\
\hline$b_{\text {oper }}$ & $\begin{array}{c}\text { unit size-dependent operating cost per } \\
\text { vehicle-hour }\end{array}$ \\
\hline$c_{\mathrm{cptl}}$ & unit fixed capital cost per vehicle-hour \\
\hline$b_{\mathrm{cptl}}$ & $\begin{array}{l}\text { unit size-dependent capital cost per } \\
\text { vehicle-hour }\end{array}$ \\
\hline$\eta^{\mathrm{sa}}$ & $\begin{array}{l}\text { reduced fixed unit operating cost of } \\
\text { semi-auton. vs. conv. bus }\end{array}$ \\
\hline$\eta^{\mathrm{fa}}$ & $\begin{array}{l}\text { reduced fixed unit operating cost of fully } \\
\text { auton. vs. conv. bus }\end{array}$ \\
\hline$\beta^{\mathrm{sa}}$ & $\begin{array}{l}\text { additional fixed unit capital cost of } \\
\text { semi-auton. vs. conv. bus }\end{array}$ \\
\hline$\beta^{\mathrm{fa}}$ & $\begin{array}{l}\text { additional fixed unit capital cost of fully } \\
\text { auton. vs. conv. bus }\end{array}$ \\
\hline$\phi^{\mathrm{sa}}$ & $\begin{array}{l}\text { relative commercial speed of semi-auton. } \\
\text { vs. conv. bus }\end{array}$ \\
\hline$\phi^{\mathrm{fa}}$ & $\begin{array}{l}\text { relative commercial speed of fully auton. } \\
\text { vs. conv. bus }\end{array}$ \\
\hline$t_{\text {corr }}$ & $\begin{array}{c}\text { nominal one-way travel time on the } \\
\text { corridor }\end{array}$ \\
\hline$t_{\text {bran }}$ & $\begin{array}{l}\text { nominal one-way travel time on the } \\
\text { branch }\end{array}$ \\
\hline$t_{\text {rnd }}$ & nominal round-trip travel time \\
\hline$q_{\text {corr }}$ & directional travel demand on the corridor \\
\hline$q_{\text {full }}$ & $\begin{array}{l}\text { directional travel demand on between } \\
\text { corridor and each branch }\end{array}$ \\
\hline$q_{\text {bran }}$ & directional travel demand on each branch \\
\hline$q_{\text {wait }}$ & $\begin{array}{l}\text { total waiting time per bus line per unit } \\
\text { headway }\end{array}$ \\
\hline$q_{\max }$ & maximum load of each bus line \\
\hline$\gamma$ & desired occupancy rate \\
\hline$p_{r}$ & platooning rate \\
\hline
\end{tabular}

parameters $b_{\text {oper }}$ and $c_{\text {oper }}$ related to the marginal and fixed unit costs, respectively,

$$
g_{\text {oper }}^{c}=c_{\text {oper }}+b_{\text {oper }} s^{c} .
$$




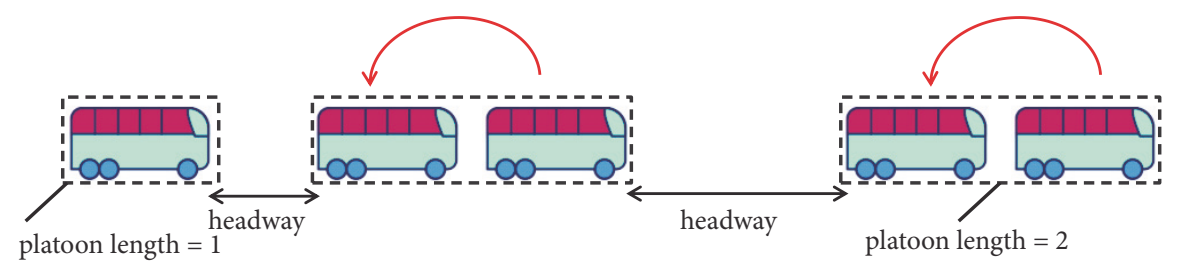

FIGURE 1: Illustration of bus platoons. The arrows indicate the vehicle-to-vehicle (V2V) wireless communication.

The unit capital cost per vehicle, normalized on an hourly basis, is also a linear function of vehicle size,

$$
g_{\mathrm{cptl}}^{\mathrm{c}}=c_{\mathrm{cptl}}+b_{\mathrm{cptl}} s^{\mathrm{c}}
$$

where parameters $b_{\mathrm{cptl}}$ and $c_{\mathrm{cptl}}$ represent the marginal and fixed unit capital costs, respectively.

2.2. Impacts of Automation. A major benefit brought by automation technology is the reduction or elimination of the on-vehicle crew cost, which can be represented by a reduction coefficient in front of the fixed unit operating $\operatorname{cost} c_{\text {oper }}$. Other impacts such as the change in fuel use and tire cost are limited compared to the labor savings and are neglected in this analysis. In addition, many factors contribute to fuel use and energy efficiency, which may require careful research on the quantification of these benefits [22].

The operating procedure of cooperative bus platooning with semi-autonomous buses is illustrated in Figure 1. By forming bus platoons, all buses except for the leading one experience operating cost savings due to the reduced cost of labor. Thus,

$$
g_{\mathrm{oper}}^{\mathrm{sa}}= \begin{cases}c_{\mathrm{oper}}+b_{\mathrm{oper}} s^{\mathrm{sa}} & \text { platoon leader } \\ \left(1-\eta^{\mathrm{sa}}\right) c_{\mathrm{oper}}+b_{\mathrm{oper}} s^{\mathrm{sa}} & \text { platoon follower }\end{cases}
$$

where $\eta^{\text {sa }}$ is the fixed unit operating cost reduction coefficient, $0<\eta^{\text {sa }}<1$. Meanwhile, automation technology is expected to increase the capital cost because additional devices or modules (e.g., LIDAR) will be required. This is represented by an fixed unit operating cost increase parameter $\beta^{\text {sa }}>0$.

$$
g_{\mathrm{cptl}}^{\mathrm{sa}}=\left(1+\beta^{\mathrm{sa}}\right) c_{\mathrm{cptl}}+b_{\mathrm{cptl}} s^{\mathrm{sa}}
$$

Note that the marginal impact of vehicle size on operating and capital cost is assumed to be the same as for conventional buses.

For fully autonomous buses, the on-vehicle crew cost is assumed to be eliminated completely since drivers are not needed, which results in lower hourly operating cost per bus. The unit cost is

$$
g_{\mathrm{oper}}^{\mathrm{fa}}=\left(1-\eta^{\mathrm{fa}}\right) c_{\mathrm{oper}}+b_{\mathrm{oper}} s^{\mathrm{fa}},
$$

where $\eta^{\mathrm{fa}}$ is the fixed unit capital cost reduction coefficient, $0<\eta^{\mathrm{fa}}<1$. It is assumed that the cost reduction of full automation is equal or greater than the cost reduction of semi-automation, i.e., $\eta^{\mathrm{fa}} \geq \eta^{\text {sa }}$. Further, full automation is expected to increase the fixed unit capital cost by a factor $\beta^{\mathrm{fa}}$, equal to or greater than that for semi-automation,

$$
g_{\mathrm{cptl}}^{\mathrm{fa}}=\left(1+\beta^{\mathrm{fa}}\right) c_{\mathrm{cptl}}+b_{\mathrm{cptl}} s^{\mathrm{fa}},
$$

where $\beta^{\mathrm{fa}} \geq \beta^{\text {sa }}>0$. The marginal impact of vehicle size on operating and capital cost is assumed to be the same as for semi-autonomous and conventional buses.

The commercial speed of buses in urban areas is determined by several factors, including attributes of the bus and the traffic conditions. For conventional buses, a commonly used value is $15 \mathrm{~km} /$ hour. For autonomous buses, the commercial speed may be higher or lower than for conventional buses, depending on when and where the buses are adopted. Currently, fully autonomous buses in pilot platforms are operated in a conservative way so as to avoid any collision with pedestrians, cyclists, or cars when running in mixed traffic. Some trials are carried out on segregated lanes without interacting with other traffic components and the speed can be higher than the nominal speed. Although drivers do not operate these buses, essentially, these trials are not fully autonomous so they are not considered in our study. Therefore, the driving speed of fully autonomous buses may be lower than the nominal speed in the near future. As the technology develops however, if the infrastructure and the surrounding traffic also favor automation, traffic conditions may improve so that autonomous buses can drive at the same speed as conventional buses. In addition, platooning with semi-autonomous buses requires coordination so that they can drive simultaneously, which may take time. For these reasons, we consider the relative speeds of semi-autonomous and fully autonomous buses with respect to the nominal speed, which are denoted by $\phi^{\text {sa }}$ and $\phi^{\mathrm{fa}}$, respectively, where $\phi^{\mathrm{sa}}>0, \phi^{\mathrm{fa}}>0$. As a baseline, we assume that the commercial speed is independent of vehicle technology, i.e., $\phi^{\mathrm{sa}}=1, \phi^{\mathrm{fa}}=1$.

\section{Bus Operation Modeling}

In this section we formulate the optimal service provision problem given conventional, semi-autonomous, or fully autonomous buses. The generalized cost function includes passengers' waiting cost and on-board riding time cost, and the service provider's operating and capital costs.

3.1. Network Structure. The analysis is based on the network shown in Figure 2, which is composed of a common corridor $\mathrm{AB}$ and $m$ branches $\mathrm{BC}, \mathrm{BD}$, etc. This structure is analogous to 


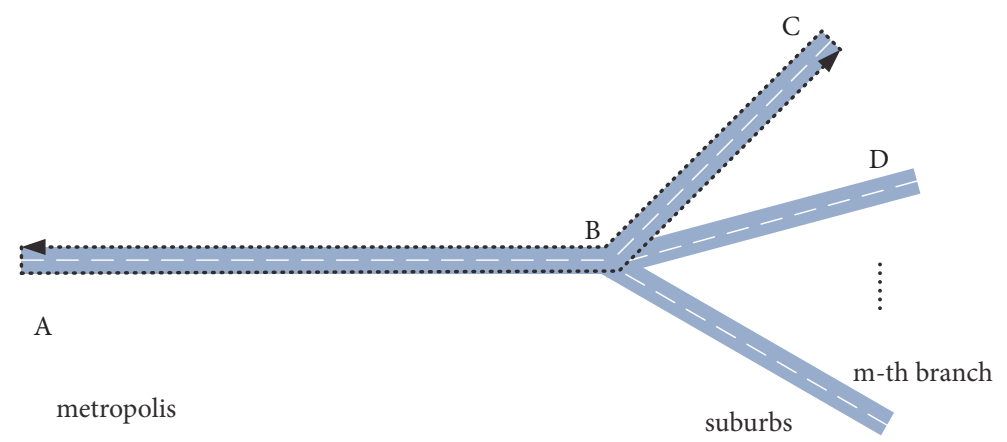

FIGURE 2: A trunk-and-branches public transport network connecting metropolis and suburbs. The dotted line with arrows shows the operational strategy for one round trip traversed by one of the bus lines. The same strategy applies to all $m$ bus lines in the transit network.

typical networks connecting the metropolis and the suburbs of an urban area. The road conditions in this setting are less complicated than in central urban areas (streets, traffic signals, etc.), which makes vehicle platooning feasible. Each branch is served by a specific bus line, where each line starts at the city center $\mathrm{A}$, drives along the common corridor $\mathrm{AB}$, reaches the terminal of a branch in the suburb ( $\mathrm{C}$ or $\mathrm{D}$ or $\mathrm{E}$, etc.), and then returns to the city center in the opposite direction (e.g., $\mathrm{A} \longrightarrow \mathrm{B} \longrightarrow \mathrm{C} \longrightarrow \mathrm{B} \longrightarrow \mathrm{A}$ ).

The $m$ branches are assumed to be symmetric in both service and demand. The $m$ bus lines have the same headway between consecutive buses, denoted by $h$. Driving times are static and deterministic, and we ignore acceleration time and deceleration time. The dwell time is insignificant compared to the driving time and is not considered. The travel time on $\mathrm{AB}$ is denoted by $t_{\text {corr }}$, while the travel time on each branch $\mathrm{BC}$, $\mathrm{BD}$, etc. is denoted by $t_{\mathrm{bran}}$. The full round-trip travel time is $t_{\text {rnd }}=2\left(t_{\text {corr }}+t_{\text {bran }}\right)$.

Travel demand in the network is static and deterministic but can vary between different parts of the network. Demand is divided into three categories: (1) trips along the corridor only, (2) trips along each branch, and (3) trips between the corridor and the branch. While travelers along the branches or between the corridor and the branches must wait for a specific line, travelers along the corridor are indifferent to the different lines and board the first arriving bus from any line.

Demand is assumed to follow a continuous uniform distribution along the corridor and branches; for each category of trips, the demand density between any two points is constant. The total travel demand per unit time along the corridor is denoted by $q_{\text {corr }}$, and the total demand along each branch (BC, BD, etc.) is denoted by $q_{\text {bran }}$, while the total demand between the corridor and each branch (AC, AD, etc.) is denoted by $q_{\text {full }}$.

3.2. Service Frequency. In this study, service frequency is determined based on the maximum load method, where the headway is set to serve the largest load along the line, Ceder [23]. For any bus line, the total directional demand on the corridor and the branch are $q_{\text {corr }} / m+q_{\text {full }}$ and $q_{\text {full }}+$ $q_{\text {bran }}$, respectively. It is likely that demand in the between the metropolis and the suburb is larger than within the suburb, so we assume that the maximum load always appears on the corridor AB. Appendix A shows that the maximum load is given by $q_{\max }=\left(m q_{\text {full }}+2 q_{\text {corr }}\right)^{2} /\left(8 m q_{\text {corr }}\right)$. Therefore, the service headway is calculated by

$$
h=\frac{\gamma s}{q_{\max }}
$$

where $\gamma$ is the desired bus occupancy rate, $0<\gamma \leq 1$. The headway is thus proportional to the vehicle size. The minimum fleet size required to serve each line given roundtrip travel time $t_{\text {rnd }}$ and headway $h$ is

$$
n=\frac{t_{\mathrm{rnd}}}{h}=\frac{q_{\mathrm{max}} t_{\mathrm{rnd}}}{\gamma s},
$$

inversely proportional to the vehicle size.

3.3. Objective Function. The objective function to minimize is the total cost $C_{\text {tot }}$, which consists of four parts: waiting cost $C_{\text {wait }}$, on-board riding cost $C_{\text {ride }}$, operating cost $C_{\text {oper }}$, and capital cost $C_{\mathrm{cptl}}$ :

$$
C_{\text {tot }}=C_{\text {wait }}+C_{\text {ride }}+C_{\text {oper }}+C_{\text {cptl }} \text {. }
$$

In the next subsections, the different cost components are derived for operations with each of the three vehicle technologies. It is assumed that for a given type of vehicle, every bus has the same parameters, including capacity and capital cost.

3.4. Conventional Buses. When the $m$ lines are operated with conventional buses, buses from different lines traverse the corridor $\mathrm{AB}$ successively with the same headway $h^{\mathrm{c}}$. The combined headway on the corridor is thus $h^{\mathrm{c}} / \mathrm{m}$. The waiting cost is calculated based on the value of waiting time $c_{\text {wait }}$, the average waiting time per passenger, which is assumed to be half of the headway, and the demand. The average waiting time for passengers that travel only on the corridor is $h^{\mathrm{c}} /(2 m)$, while the average waiting time for passengers that travel on the branch or on the full line is $h^{\mathrm{c}} / 2$. The total hourly waiting cost is

$$
C_{\text {wait }}^{c}=m c_{\text {wait }} q_{\text {wait }}^{c} h^{c},
$$

where $q_{\text {wait }}^{\mathrm{c}}=q_{\text {corr }} / m^{2}+q_{\text {full }}+q_{\text {bran }}$ represents the total waiting time per line arising from one unit of headway time. 
The total hourly riding cost is the product of the value of in-vehicle time $c_{\text {ride }}$ and the total travel time, which is derived in Appendix B from the total riding distance and the commercial speed,

$$
\begin{aligned}
& C_{\text {ride }}^{\mathrm{c}}=\frac{c_{\text {ride }}}{3}\left[2 q_{\text {corr }} t_{\text {corr }}+3 m q_{\text {full }}\left(t_{\text {corr }}+t_{\text {bran }}\right)\right. \\
& \left.\quad+2 m q_{\text {bran }} t_{\text {bran }}\right] .
\end{aligned}
$$

Note that the riding cost is not influenced by the vehicle size or headway. The total hourly operating cost is given by the bus fleet size, which is determined by the headway and the round-trip travel time according to (8), multiplied by the unit operating cost,

$$
C_{\text {oper }}^{\mathrm{c}}=\frac{m\left(c_{\text {oper }}+b_{\text {oper }} s^{\mathrm{c}}\right) t_{\mathrm{rnd}}}{h^{\mathrm{c}}} .
$$

Similarly, the total hourly capital cost is given by the bus fleet size multiplied by the unit capital cost,

$$
C_{\mathrm{cptl}}^{\mathrm{c}}=\frac{m\left(c_{\mathrm{cptl}}+b_{\mathrm{cptl}} s^{\mathrm{c}}\right) t_{\mathrm{rnd}}}{h^{\mathrm{c}}} .
$$

By substituting the relation between vehicle size and headway (7) into (10)-(13), the total cost $C_{\text {tot }}^{c}$ is expressed as a function of vehicle size $s^{c}$ :

$$
\begin{aligned}
C_{\text {tot }}^{\mathrm{c}}= & \frac{m \gamma c_{\text {wait }} q_{\text {wait }}^{\mathrm{c}} s^{\mathrm{c}}+\frac{m\left(c_{\mathrm{oper}}+c_{\mathrm{cptl}}\right) q_{\text {max }} t_{\mathrm{rnd}}}{\gamma s^{\mathrm{c}}}}{q_{\max }} \\
& +\frac{m\left(b_{\mathrm{oper}}+b_{\mathrm{cptl}}\right) q_{\text {max }} t_{\mathrm{rnd}}}{\gamma}+C_{\text {ride }}^{\mathrm{c}} .
\end{aligned}
$$

As can be seen, minimizing waiting times on the one hand and operating and capital costs on the other hand are conflicting goals. The optimal vehicle size is obtained from the first-order condition of $C_{\text {tot }}^{c}$ with respect to the vehicle size $s^{\mathrm{c}}$ :

$$
s^{\mathrm{c} *}=\frac{q_{\mathrm{max}}}{\gamma} \sqrt{\frac{\left(c_{\mathrm{oper}}+c_{\mathrm{cptl}}\right) t_{\mathrm{rnd}}}{c_{\text {wait }} q_{\mathrm{wait}}^{\mathrm{c}}}} .
$$

This well-known square root formula shows that the optimal vehicle size increases with the service provider's fixed operating and capital costs, since higher costs encourage larger and fewer vehicles. Meanwhile, the vehicle size decreases with the passengers' value of waiting time, since smaller vehicles imply shorter waiting times. The optimal headway and optimal fleet size are readily obtained by substituting (15) into (7) and (8), respectively. Substituting (15) into (14), the minimum total cost is

$$
\begin{aligned}
C_{\text {tot }}^{\mathrm{c} *}= & 2 m \sqrt{\left(c_{\text {oper }}+c_{\text {cptl }}\right) c_{\text {wait }} q_{\text {wait }}^{\mathrm{c}} t_{\text {rnd }}} \\
+ & \frac{m\left(b_{\text {oper }}+b_{\text {cptl }}\right) q_{\text {max }} t_{\text {rnd }}}{\gamma}+C_{\text {ride }}^{\mathrm{c}}
\end{aligned}
$$

The first term is the sum of passengers' waiting cost and a baseline operating and capital cost that is independent of vehicle size, which are equal in magnitude. The second term represents the additional operating and capital cost of the vehicle capacity that is required to serve the travel demand. The third term is the passengers' riding cost.

3.5. Fully Autonomous Buses. For a given headway $h^{\mathrm{fa}}$, waiting cost for fully autonomous buses is the same as for conventional buses and is given by (10), replacing $h^{\mathrm{c}}$ with $h^{\mathrm{fa}}$. Meanwhile, as discussed in Section 2.2, the driving speed of fully autonomous buses may differ from conventional buses by a factor $\phi^{\mathrm{fa}}>0$. This implies that the total riding cost differs by the same factor, $C_{\text {ride }}^{\mathrm{fa}}=C_{\text {ride }}^{\mathrm{c}} / \phi^{\mathrm{fa}}$.

By eliminating the need for drivers, fully autonomous buses have a lower fixed hourly operating cost per bus than conventional buses by a factor $\eta^{\mathrm{fa}}$. Meanwhile, the round-trip travel time changes from $t_{\text {rnd }}$ to $t_{\text {rnd }} / \phi^{\mathrm{fa}}$, which means that more vehicles are needed to operate with a given headway if $\phi^{\mathrm{fa}}<1$. The total hourly operating and capital costs are

$$
\begin{aligned}
C_{\text {oper }}^{\mathrm{fa}} & =\frac{m\left[\left(1-\eta^{\mathrm{fa}}\right) c_{\text {oper }}+b_{\text {oper }} s^{\mathrm{fa}}\right] t_{\text {rnd }}}{\phi^{\mathrm{fa}} h^{\mathrm{fa}}} \\
C_{\mathrm{cptl}}^{\mathrm{fa}} & =\frac{m\left[\left(1+\beta^{\mathrm{fa}}\right) c_{\mathrm{cptl}}+b_{\mathrm{cptl}} s^{\mathrm{fa}}\right] t_{\text {rnd }}}{\phi^{\mathrm{fa}} h^{\mathrm{fa}}} .
\end{aligned}
$$

By applying the vehicle size-headway relationship (7), the total cost $C_{\text {tot }}^{\mathrm{fa}}$ is expressed as a function of vehicle size. As before, the optimal vehicle size is obtained from the firstorder optimality conditions of the total cost,

$$
s^{\mathrm{fa} *}=\frac{q_{\max }}{\gamma} \sqrt{\frac{\left[\left(1-\eta^{\mathrm{fa}}\right) c_{\text {oper }}+\left(1+\beta^{\mathrm{fa}}\right) c_{\mathrm{cptl}}\right] t_{\mathrm{rnd}}}{\phi^{\mathrm{fa}} c_{\text {wait }} q_{\text {wait }}^{\mathrm{c}}}} .
$$

As for semi-autonomous buses, the optimal vehicle size decreases with the reduced operating cost, but increases with the additional capital cost and the possibly lower commercial speed. The minimum total cost is

$$
\begin{aligned}
& C_{\text {tot }}^{\mathrm{fa} *} \\
& =2 m \sqrt{\frac{\left[\left(1-\eta^{\mathrm{fa}}\right) c_{\text {oper }}+\left(1+\beta^{\mathrm{fa}}\right) c_{\text {cptl }}\right] c_{\text {wait }} q_{\text {wait }}^{\mathrm{c}} t_{\text {rnd }}}{\phi^{\mathrm{fa}}}} \\
& +\frac{m\left(b_{\text {oper }}+b_{\text {cptl }}\right) q_{\text {max }} t_{\text {rnd }}}{\gamma \phi^{\mathrm{fa}}}+\frac{C_{\text {ride }}^{\mathrm{c}}}{\phi^{\mathrm{fa}}} .
\end{aligned}
$$

3.6. Semi-Autonomous Buses. Without loss of generality, we consider the scenario where $m$ bus lines are grouped together into $r\left(r \in \mathbb{Z}_{>0}, 1 \leq r \leq m\right)$ platoons on the corridor and then split up into individual branches. If $r=1$, buses from all lines drive together on the corridor; in the other extreme, if $r=m$, every bus drives individually and no operating cost savings are incurred. For every platoon $i, 1 \leq i \leq r$, the number of 
members, also termed platoon length, is denoted by $m_{i}$. The total length of all platoons must equal the number of lines: $m_{1}+m_{2}+\ldots+m_{r}=m$.

To facilitate the further analysis, we define the concept platooning rate, which is the proportion of the time consumed by all platoon followers with respect to the total travel time. With $r$ platoons, the platooning rate $p_{r}$ is computed as

$$
p_{r}=\frac{2(m-r) t_{\text {corr }}}{m t_{\text {rnd }}} \text {. }
$$

It is assumed here that the headway of platoon $i$ is proportional to its length $m_{i}$, as indicated in Figure 1. Specifically, the headway of platoon $i$ is $m_{i} h^{\mathrm{sa}} / m$. This implies that the maximum load $q_{\max }$ is still given by (7). The average waiting time for platoon $i$ on the corridor is $m_{i} h^{\text {sa }} /(2 m)$, while it is still $h^{\text {sa }} / 2$ on the branch. More specifically, the waiting time for the directional demands $q_{\text {full }}$ and $q_{\text {bran }}$ is not affected by platooning since they have to wait for a certain bus line (of which the headway is always $h^{\text {sa }}$ ), whereas the waiting time for a specific corridor traveler in $q_{\text {corr }}$ depends on which platoon the traveler boards (whichever comes first), regardless of the trip direction. What matters to the corridor travelers is the platoon configuration, i.e., how many platoons are formed and the respective number of members in each platoon. We assume the extra time required to form platoons is short enough to be ignored and the platoon configuration does not change in the departing and returning trips. Therefore, the total waiting time for inward trips (from C to A or from B to A) and outward trips will be the same. The waiting time cost is

$$
C_{\text {wait }}^{\mathrm{sa}}=m c_{\text {wait }} q_{\text {wait }}^{\mathrm{sa}} h^{\mathrm{sa}},
$$

where $q_{\text {wait }}^{\text {sa }}=\left(m_{1}^{2}+m_{2}^{2}+\ldots+m_{r}^{2}\right) q_{\text {corr }} / m^{3}+q_{\text {full }}+q_{\text {bran }}$ represents the total waiting time per line corresponding to one unit of headway time.

As discussed in Section 2.2, the commercial speed of semi-autonomous buses may differ from the nominal speed of conventional buses by a factor $\phi^{\text {sa }}>0$. The riding cost of semi-autonomous buses is thus divided by $\phi^{\mathrm{sa}}, C_{\text {ride }}^{\mathrm{sa}}=$ $C_{\text {ride }}^{\mathrm{c}} / \phi^{\text {sa }}$. As for conventional buses, the hourly operating cost is given by the bus fleet size multiplied by the unit operating cost. The fixed operating cost for each follower bus is reduced by a factor $\eta^{\text {sa }}$ when driving on the corridor, so that the total cost reduction per hour is $\eta^{\mathrm{sa}} p_{r}$,

$$
C_{\mathrm{oper}}^{\mathrm{sa}}=\frac{m\left[\left(1-\eta^{\mathrm{sa}} p_{r}\right) c_{\mathrm{oper}}+b_{\mathrm{oper}} s^{\mathrm{sa}}\right] t_{\mathrm{rnd}}}{\phi^{\mathrm{sa}} h^{\mathrm{sa}}} .
$$

Similarly, the fixed capital cost for each vehicle is increased by a factor $\beta^{\text {sa }}$ compared to conventional buses,

$$
C_{\mathrm{cptl}}^{\mathrm{sa}}=\frac{m\left[\left(1+\beta^{\mathrm{sa}}\right) c_{\mathrm{cptl}}+b_{\mathrm{cptl}} s^{\mathrm{sa}}\right] t_{\mathrm{rnd}}}{\phi^{\mathrm{sa}} h^{\mathrm{sa}}} .
$$

By applying the relation between vehicle size and headway (7) to (22), (23), and (24), the total cost $C_{\text {tot }}^{\text {sa }}$ is formulated as a function of vehicle size, and the optimal vehicle size $s^{\text {sa* }}$ is obtained from the first-order condition of $C_{\text {tot }}^{\text {sa }}$ with respect to $s^{\mathrm{sa}}$,

$$
s^{\mathrm{sa} *}=\frac{q_{\max }}{\gamma} \sqrt{\frac{\left[\left(1-\eta^{\mathrm{sa}} p_{r}\right) c_{\mathrm{oper}}+\left(1+\beta^{\mathrm{sa}}\right) c_{\mathrm{cpt}}\right] t_{\mathrm{rnd}}}{\phi^{\mathrm{sa}} c_{\text {wait }} q_{\text {wait }}^{\mathrm{sa}}}} .
$$

The optimal vehicle size thus decreases with the reduced operating cost for platoon followers, which is proportional to the platooning rate. Meanwhile, the size increases with the additional capital cost and the possibly lower commercial speed. The minimum total cost is

$$
\begin{aligned}
& C_{\text {tot }}^{\text {sa* }} \\
& =2 m \sqrt{\frac{\left[\left(1-\eta^{\text {sa }} p_{r}\right) c_{\text {oper }}+\left(1+\beta^{\text {sa }}\right) c_{\text {cpt }}\right] c_{\text {wait }} q_{\text {wait }}^{\text {sa }} t_{\text {rnd }}}{\phi^{\text {sa }}}} \\
& +\frac{m\left(b_{\text {oper }}+b_{\text {cptl }}\right) q_{\text {max }} t_{\text {rnd }}}{\gamma \phi^{\text {sa }}}+\frac{C_{\text {ride }}^{\mathrm{c}}}{\phi^{\text {sa }}} .
\end{aligned}
$$

Compared to conventional buses, the relative speed of semiautonomous vehicles influences all terms of the total cost negatively if $\phi^{\text {sa }}<1$.

Longer platoons reduce operating cost but increase waiting cost on the corridor, and part of the service design is to determine the optimal platoon configuration. If this discrete combinatorial optimization problem is relaxed to a continuous problem, the symmetry of the network implies that the optimal configuration for a given number of platoons $r_{\text {relax }}\left(r_{\text {relax }} \in \mathbb{R}_{>0}, 1 \leq r_{\text {relax }} \leq m\right)$ occurs when all platoons have equal length. If we set

$$
q_{\text {wait }}^{\text {sa }}=\frac{1}{m r_{\text {relax }}} q_{\text {corr }}+q_{\text {full }}+q_{\text {bran }}
$$

by adopting the optimum condition that $m_{1}=m_{2}=\ldots=$ $m_{r}=m / r_{\text {relax }}$, the optimal $r_{\text {relax }}$ can be obtained by numerical solution methods. Thus, the headways between consecutive platoons on the corridor are the same and the combined headway is $h^{\text {sa }} / r_{\text {relax }}$. This $\left\lfloor r_{\text {relax }}\right\rfloor$ (or $\left\lceil r_{\text {relax }}\right\rceil$ ) can be used as an approximation of $r$. Numerical analysis of the optimal platoon size is presented in Section 5.

\section{Comparison of Vehicle Technologies}

In this section, the costs and benefits of the conventional, semi-autonomous, and fully autonomous bus services are compared given that the service is optimized for each type of vehicle according to the previous section.

4.1. Impacts of Fully Autonomous Buses. The optimal size of autonomous buses is smaller than for conventional buses if and only if the net reduction in fixed unit operating and capital cost compensates for the lower commercial speed, i.e.,

$$
\frac{\left(1-\eta^{\mathrm{fa}}\right) c_{\text {oper }}+\left(1+\beta^{\mathrm{fa}}\right) c_{\mathrm{cptl}}}{c_{\text {oper }}+c_{\text {cptl }}}<\phi^{\mathrm{fa}} .
$$


The same condition applies to headways as well as the average waiting times on the corridor, the branches, and the full lines. Meanwhile, smaller vehicles imply larger fleet sizes and vice versa. The difference in total cost with fully autonomous and conventional buses is obtained from (16) and (20),

$$
\begin{aligned}
C_{\text {tot }}^{\mathrm{fa} *}- & C_{\text {tot }}^{\mathrm{c} *} \\
= & 2 m \sqrt{c_{\text {wait }} q_{\text {wait }}^{\mathrm{c}} t_{\text {rnd }}\left(c_{\text {oper }}+c_{\text {cptl }}\right)} \\
& \cdot\left(\sqrt{\frac{\left(1-\eta^{\mathrm{fa}}\right) c_{\text {oper }}+\left(1+\beta^{\mathrm{fa}}\right) c_{\mathrm{cptl}}}{\phi^{\mathrm{fa}}\left(c_{\text {oper }}+c_{\mathrm{cptl}}\right)}}-1\right) \\
+ & \left(\frac{1}{\phi^{\mathrm{fa}}}-1\right)\left[\frac{m q_{\text {max }} t_{\text {rnd }}}{\gamma}\left(b_{\text {oper }}+b_{\text {cptl }}\right)+C_{\text {ride }}^{\mathrm{c}}\right] .
\end{aligned}
$$

Equation (29) imposes a stronger condition for autonomous buses to be profitable than condition (28) for the use of smaller vehicles, as long as autonomous buses drive at lower speed than conventional buses. Thus, autonomous buses, if beneficial, will be smaller than conventional buses, require a larger vehicle fleet, and operate with a shorter headway. Passengers will benefit from shorter waiting times but incur longer in-vehicle travel times if $\phi^{\mathrm{fa}}<1$. The service provider will benefit through lower baseline costs, but possibly incur increased additional cost for serving the travel demand due to lower commercial speed. The net benefits or losses depend on the settings of the service and are studied numerically in Section 5.

Note that if $\phi^{\mathrm{fa}}=1$, condition (28) and the condition for fully autonomous buses to be beneficial both simplify to $\beta^{\mathrm{fa}} c_{\text {cptl }}<\eta^{\mathrm{fa}} c_{\text {oper }}$; i.e., the additional fixed unit capital cost should be lower than the reduction of fixed unit operating cost.

4.2. Impacts of Semi-Autonomous Buses. From (15) and (25), it follows that semi-autonomous buses will be smaller than conventional buses if the net savings in the fixed unit operating and capital cost can compensate for the additional waiting time, i.e., if and only if

$$
\frac{\left(1-\eta^{\mathrm{sa}} p_{r}\right) c_{\mathrm{oper}}+\left(1+\beta^{\mathrm{sa}}\right) c_{\mathrm{cptl}}}{c_{\text {oper }}+c_{\mathrm{cptl}}}<\frac{\phi^{\mathrm{sa}} q_{\mathrm{wait}}^{\mathrm{sa}}}{q_{\text {wait }}^{\mathrm{c}}} .
$$

Since headway is proportional to vehicle size according to (7), the service along each bus line (or on the branch) with semiautonomous vehicles is run with shorter headways than for conventional buses if (30) holds. Further, since fleet size is inversely proportional to the headway (8), the fleet size of the semi-autonomous buses will be larger than for conventional buses if and only if condition (30) applies. For passengers traveling to or from the branches, condition (30) also applies for whether waiting times are shorter with semi-autonomous buses than conventional buses. For passengers traveling only on the corridor, however, the waiting time is shorter with semi-autonomous buses if and only if

$$
\frac{\left(1-\eta^{\mathrm{sa}} p_{r}\right) c_{\mathrm{oper}}+\left(1+\beta^{\mathrm{sa}}\right) c_{\mathrm{cptl}}}{c_{\text {oper }}+c_{\mathrm{cptl}}}<\frac{r_{\text {relax }}^{2}}{m^{2}} \frac{\phi^{\mathrm{sa}} q_{\mathrm{wait}}^{\mathrm{sa}}}{q_{\mathrm{wait}}^{\mathrm{c}}} .
$$

Criterion (31) is more strict than (30) and becomes increasingly restricting as the number of branches $m$ grows or the number of platoons $r_{\text {relax }}$ decreases.

According to (16) and (26), the total cost with semiautonomous buses is lower than with conventional buses if and only if

$$
\begin{aligned}
C_{\text {tot }}^{\text {sa }}- & C_{\text {tot }}^{\mathrm{c} *} \\
= & 2 m \sqrt{c_{\text {wait }} q_{\text {wait }}^{\mathrm{c}} t_{\text {rnd }}\left(c_{\text {oper }}+c_{\text {cptl }}\right)} \\
& \cdot\left(\sqrt{\frac{\left[\left(1-\eta^{\mathrm{sa}} p_{r}\right) c_{\text {oper }}+\left(1+\beta^{\mathrm{sa}}\right) c_{\mathrm{cptl}}\right] q_{\mathrm{wait}}^{\mathrm{sa}}}{\phi^{\mathrm{sa}}\left(c_{\mathrm{oper}}+c_{\mathrm{cptl}}\right) q_{\text {wait }}^{\mathrm{c}}}}-1\right) \\
& +\left(\frac{1}{\phi^{\mathrm{sa}}}-1\right)\left[\frac{m q_{\text {max }} t_{\text {rnd }}}{\gamma}\left(b_{\text {oper }}+b_{\mathrm{cptl}}\right)+C_{\text {ride }}^{\mathrm{c}}\right]<0 .
\end{aligned}
$$

Condition (32) is stronger than condition (30) for using smaller vehicle sizes. It can be concluded that semiautonomous buses, if beneficial, will be smaller, require a larger vehicle fleet, and operate with a shorter headway than conventional buses. Given that semi-autonomous buses are beneficial for the total system cost, it is clear that the service provider is the main beneficiary through reduced operating costs on the corridor. Passengers traveling on the branches or between the corridor and the branches experience shorter waiting times, while passengers on the corridor likely experience longer waiting times; all passengers experience longer in-vehicle riding times if $\phi^{\text {sa }}<1$. The net benefits or losses for travelers and the service provider are studied numerically in Section 5.

\section{Numerical Analysis}

This section evaluates the cost and benefits of semiautonomous and fully autonomous buses relative to conventional buses under a range of scenarios related to network structure and demand profile, as well as operating and capital cost parameters.

5.1. Parameters. A network with $m=4$ branches and four lines, with each line serving the corridor and one branch, is considered as base scenario. The travel demand along the corridor, each full line, and each branch are $q_{\text {corr }}=480$, $q_{\text {full }}=40$, and $q_{\text {bran }}=50$ passengers/hour, respectively. The round trip takes $t_{\text {rnd }}=2$ hours to complete for each bus line, of which $t_{\text {corr }}=0.7$ hours are spent from A to B.

To study the impacts of different parameters, we vary the targeted parameter and keep remaining parameters unchanged. The nominal values of all parameters are given in Table 2, of which the first eight rows (from $c_{\text {ride }}$ to $\eta^{\mathrm{fa}}$ ) are obtained from Australian Transport Council [7]. We use the exchange rate $1 \mathrm{AUD}=0.63 \mathrm{EUR}$. Assume that the nominal speeds of all three types of vehicles are equal to $15 \mathrm{~km} / \mathrm{hour}$, i.e., $\phi^{\text {sa }}=1.0$ and $\phi^{\mathrm{fa}}=1.0$. We use linear regression to obtain the hourly operating cost and capital cost as a function of bus size. For replication, the data and the linear regression results are given in Appendix C. Australian Transport Council [7] 
TABLE 2: Nominal parameter values used in the numerical analysis.

\begin{tabular}{lc}
\hline Parameter & Value \\
\hline$c_{\text {ride }}$ & $5.53 € /$ passenger/hour \\
$c_{\text {wait }}$ & $7.80 € /$ passenger/hour \\
$c_{\text {oper }}$ & $32.9 € /$ vehicle/hour \\
$b_{\text {oper }}$ & $0.073 € /$ vehicle/hour \\
$c_{\text {cptl }}$ & $1.40 € /$ vehicle/hour \\
$b_{\text {cptl }}$ & $0.099 € /$ vehicle/hour \\
$\eta^{\text {sa }}$ & 0.63 \\
$\eta^{\text {fa }}$ & 0.63 \\
$\beta^{\text {sa }}$ & 0.2 \\
$\beta^{\text {fa }}$ & 0.5 \\
$\phi^{\text {sa }}$ & 1.0 \\
$\phi^{\text {fa }}$ & 1.0 \\
$q_{\text {corr }}$ & 480 passengers/hour \\
$q_{\text {full }}$ & 40 passengers/hour \\
$q_{\text {bran }}$ & 50 passengers/hour \\
$m$ & 4 \\
$\gamma$ & 0.8 \\
$t_{\text {corr }}$ & 0.7 hours \\
$t_{\text {bran }}$ & 2 hours \\
$t_{\text {rnd }}$ &
\end{tabular}

provides the annual capital cost without giving the yearly operation hours. However, it is mentioned in an example that when the number of peak vehicles is 600 , the annual operation hours are $1,800,000$. Although in our study, the peak-hour situation is not considered, it is common that a bus company prepares enough number of buses to handle this situation. On average, it is unlikely that each bus will operate a whole day. Therefore, we assume the annual operating hours for each bus to be $1800000 / 600=3000$, which is equivalent to about 8.2 hours per day. Based on the analysis, parameter values are $c_{\text {oper }}=32.9 € /$ vehicle/hour, $b_{\text {oper }}=0.073$ $€ /$ vehicle/hour, $c_{\text {cptl }}=1.40 € /$ vehicle/hour, and $b_{\text {cptl }}=0.099$ $€ /$ vehicle/hour.

Further, parameter value $\eta^{\mathrm{fa}}=0.63$ is used for the reduction of fixed operating cost of autonomous buses. According to Australian Transport Council [7], the on-vehicle crew cost is $20.79 € /$ hour, and we assume this part of the cost is eliminated completely. Note that the hourly on-vehicle crew cost is obtained by dividing the total annual crew cost by the total annual vehicle operating hours. Therefore, the work time limitations of drivers have already been considered in the numerical value. Thus, this on-vehicle crew cost may not be equivalent to one driver's hourly wage. For the operating cost of semi-autonomous buses, its reduction only occurs on the corridor, where we consider the benchmark case when $\eta^{\mathrm{sa}}=\eta^{\mathrm{fa}}$. When a group of buses, which depart from A without drivers in the platoon followers, arrive at $B$, the drivers board on these platoon followers and drive manually along the branches and back. Then these buses form a platoon and drivers leave the platoon followers, free to undertake other tasks until new buses arrive. In this sense, there is a group of drivers who only work on the branches and never on the corridor.

Currently, the values of $\beta^{\text {sa }}$ and $\beta^{\text {fa }}$ for the additional fixed capital cost of semi- and fully autonomous buses are both difficult to estimate. Moreover, data about these parameters from autonomous bus manufacturers are limited. Based on the assumption that fully autonomous buses are more advanced and require more capital cost than semiautonomous buses, as well as the prediction made in Wadud [24] and Fagnant and Kockelman [1] that the additional capital cost can be lowered by mass production, we use 0.2 and 0.5 as the nominal values of $\beta^{\text {sa }}$ and $\beta^{\mathrm{fa}}$, respectively.

5.2. Impact of Capital and Operating Costs. The capital cost of autonomous buses relative to conventional buses is highly uncertain and may decrease over time as the technology matures. A sensitivity analysis is carried out for the additional fixed unit capital cost parameters of semi-automation $\beta^{\text {sa }}$ and full automation $\beta^{\mathrm{fa}}$, respectively. For semi-autonomous buses, the results are approximations from the relaxed continuous optimization problem (Section 3.6). Numerical tests show that the gap between the approximated value and the exact solution is insignificant.

Figure 3 shows the total cost as well as the individual cost components (passenger cost, operating cost, and capital cost) as functions of $\beta^{\text {sa }}$ and $\beta^{\mathrm{fa}}$, where passenger cost is the sum of waiting cost and riding cost. With the given parameter values, the performance of fully autonomous buses is superior to both conventional buses and semi-autonomous buses, as indicated in Figure 3(a). The total hourly saving by fully autonomous buses when $\beta^{\text {fa }}=0.5$ is $€ 719$, among which $€ 360$ is from the passenger cost and $€ 414$ is from the operating cost. However, the capital cost is increased by $€ 55$. In comparison, there is no such major difference between semi-autonomous buses and conventional buses. Given the nominal value $\beta^{\text {sa }}=0.2$, the total hourly saving by semiautonomous buses is $€ 25.0$, among which $€ 12.5$ are attributed to reduced passenger cost and $€ 27.3$ to reduced operating cost, while the capital cost increases by $€ 14.8$. When $\beta^{\text {sa }}>0.8$, semi-autonomous buses become disadvantageous, indicating that the positive effects brought by the reduced operating cost cannot make up for the loss in passenger cost and capital cost.

Generally, Figures 3(b)-3(d) indicate that automation has the potential to reduce the operating cost of the service provider, at the cost of additional capital investment. From the service provider's perspective, when $\beta^{\text {sa }}=0.2$ and $\beta^{\mathrm{fa}}=0.5$, the hourly saving (operation and capital) can be up to $€ 12.5$ and $€ 360$ for semi-autonomous buses and fully autonomous buses, respectively. From the passengers' perspective, the reduction in labor cost allows for larger fleet sizes and shorter headways compared to conventional buses, which benefit all travelers when fully autonomous buses are adopted. However, as mentioned in Section 4.2, passengers traveling on the corridor experience longer waiting time due to the semi-autonomous buses platooning strategy, although travelers on the branches gain benefits. Both size and headway increase as $\beta^{\text {sa }}$ and $\beta^{\text {fa }}$ increase. Regardless of the cost parameters, the optimal size is largest for conventional buses 


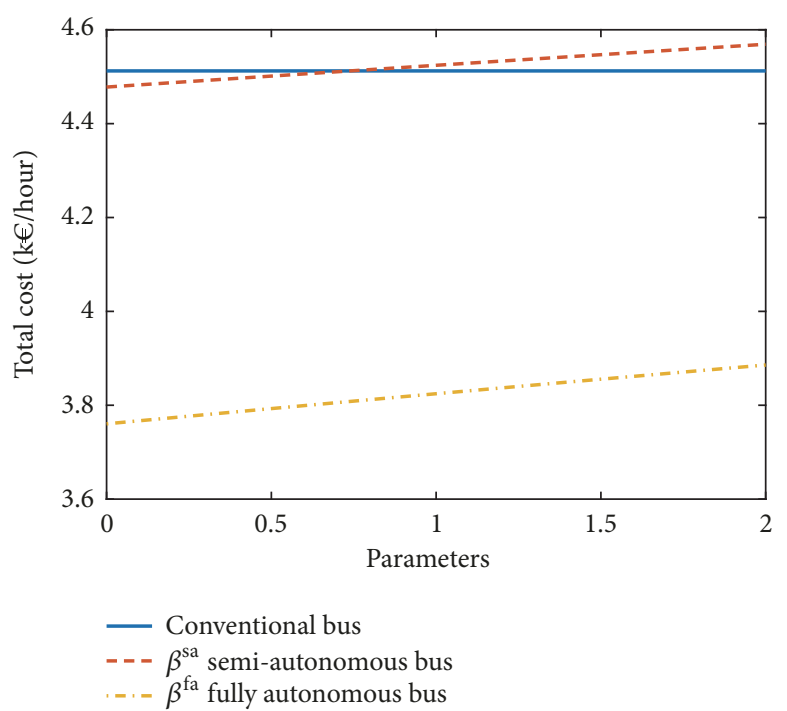

(a)

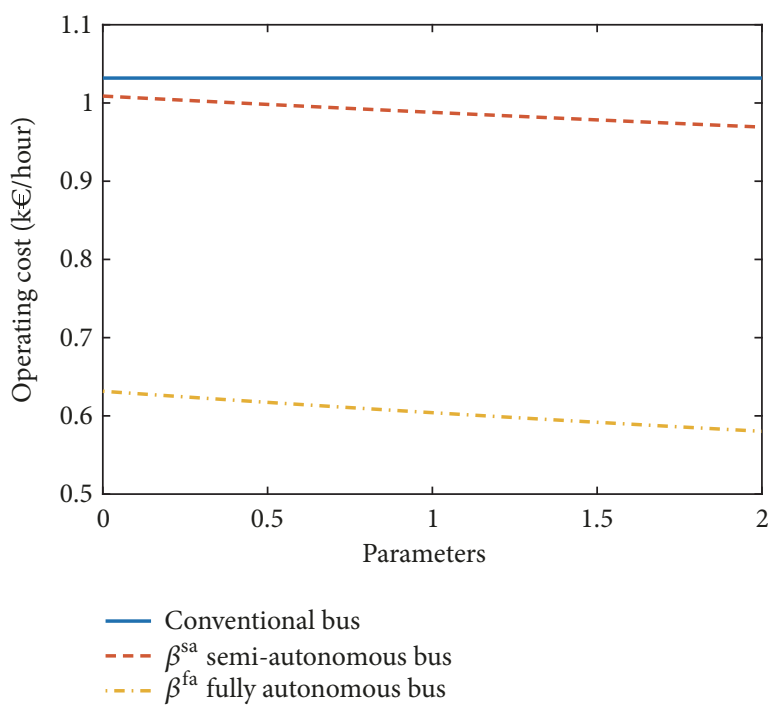

(c)

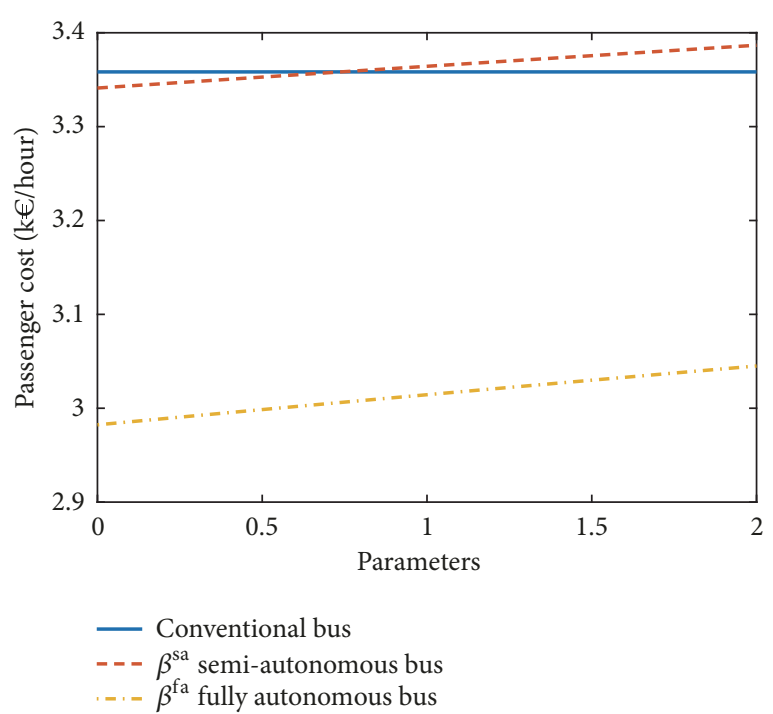

(b)

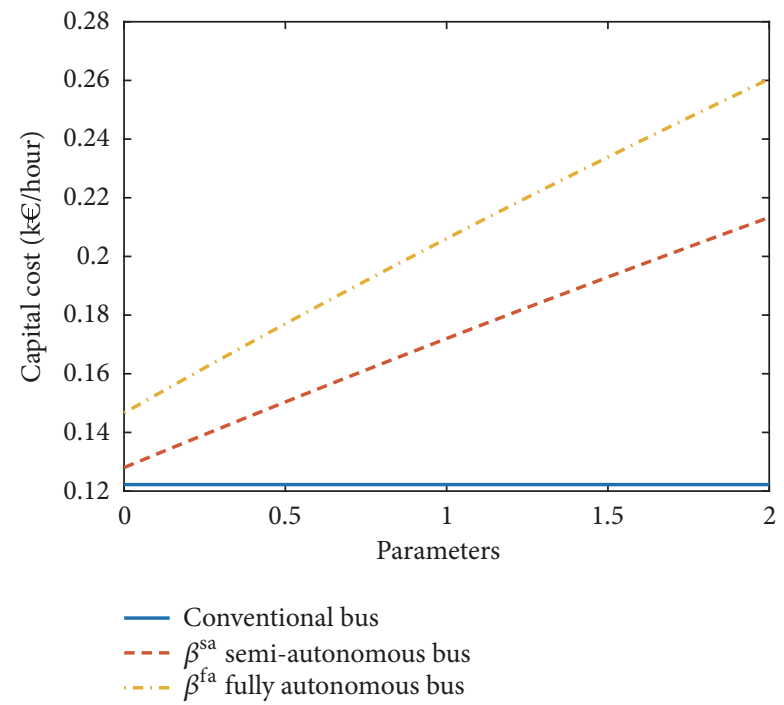

(d)

FIGURE 3: (a) Total cost, (b) passenger cost, (c) operating cost, and (d) capital cost as functions of parameters $\beta^{\text {sa }}$ and $\beta^{\text {fa }}$. Other parameters according to Table 2 .

and smallest for fully autonomous buses. Semi-autonomous buses tend to form longer platoons as $\eta^{\text {sa }}$ increases and $\beta^{\text {sa }}$ decreases.

Figures 4(a)-4(b) show the preferable regions of conventional buses, semi-autonomous buses, and fully autonomous buses with respect to the additional fixed unit capital and reduced fixed unit operating costs $\left(\beta^{\text {sa }}\right.$ and $\eta^{\text {sa }}$ for semiautonomous buses, $\beta^{\text {fa }}$ and $\eta^{\text {fa }}$ for fully autonomous buses). Hence, the result in this figure is of particular interest for one of the key issues of this paper: whether and when the benefits brought by the automation technology could outweigh the induced negative effects caused by the extra capital cost. Although the benefits exist not only in the operating cost, which is the direct result of the labor savings, the tradeoffs among all cost components and interrelated variables are driven by the pair of $\beta^{\mathrm{sa}}$ and $\eta^{\mathrm{sa}}\left(\beta^{\mathrm{fa}}\right.$ and $\left.\eta^{\mathrm{fa}}\right)$. It can be seen that for semi-autonomous buses to be beneficial when $\beta^{\text {sa }}=0.2$, the unit operating cost reduction is not required to be up to the nominal value 0.63 , but $\eta^{\text {sa }} \geq 0.499$ will suffice. Meanwhile, for fully autonomous buses to be preferable when $\beta^{\mathrm{fa}}=0.5$, the reduction of the unit operating cost should satisfy $\eta^{\mathrm{fa}} \geq 0.022$, which is far below the upper bound of $\eta^{\mathrm{fa}}$.

Semi-autonomous buses require a certain degree of operating cost reduction to be more efficient than conventional bus, even if the capital costs of them are the same (i.e., $\beta^{\text {sa }}=$ 0 ). This is because the additional waiting cost incurred on the corridor needs to be compensated, but the waiting cost savings on the branches are not enough. However, as $\beta^{\mathrm{fa}}$ approaches 0 in Figure 4(b), fully autonomous buses become 


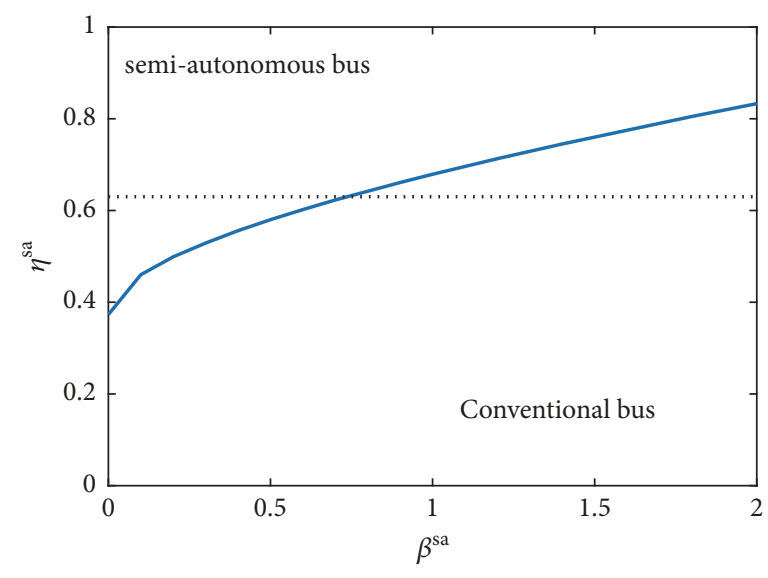

(a)

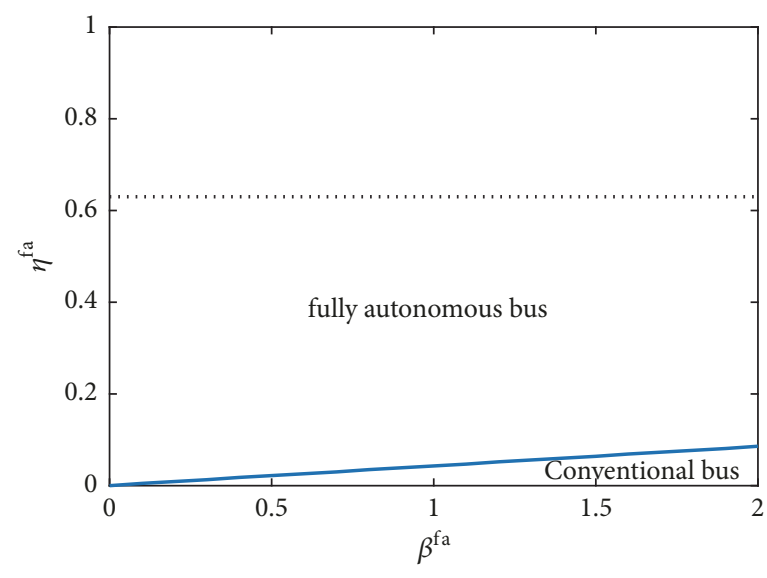

(b)

Figure 4: Applicability of (a) semi-autonomous buses and (b) fully autonomous buses, as functions of additional fixed unit capital and reduced operating costs $\beta^{\mathrm{sa}}, \eta^{\mathrm{sa}}, \beta^{\mathrm{fa}}, \eta^{\mathrm{fa}}$. The horizontal dashed line indicates the upper bound 0.63 . Other parameters according to Table 2 .

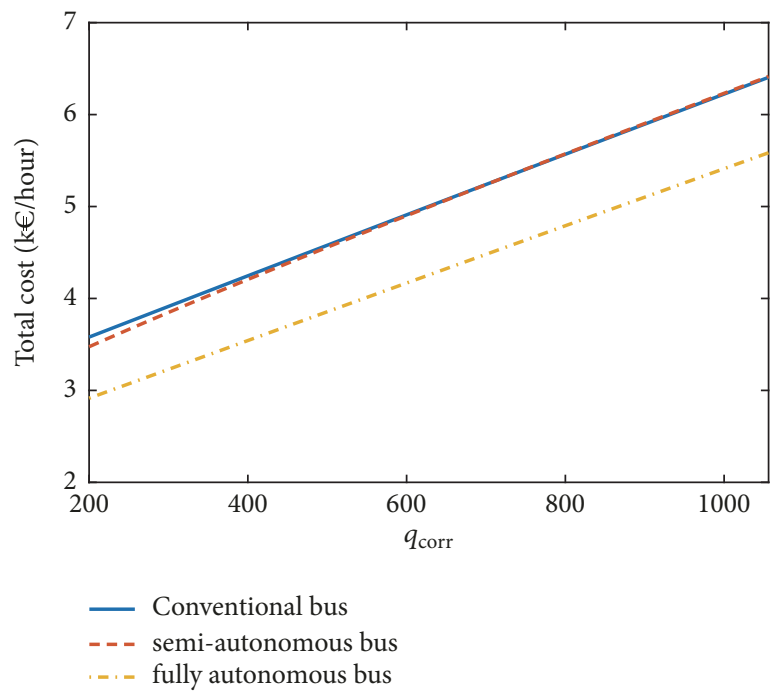

(a)

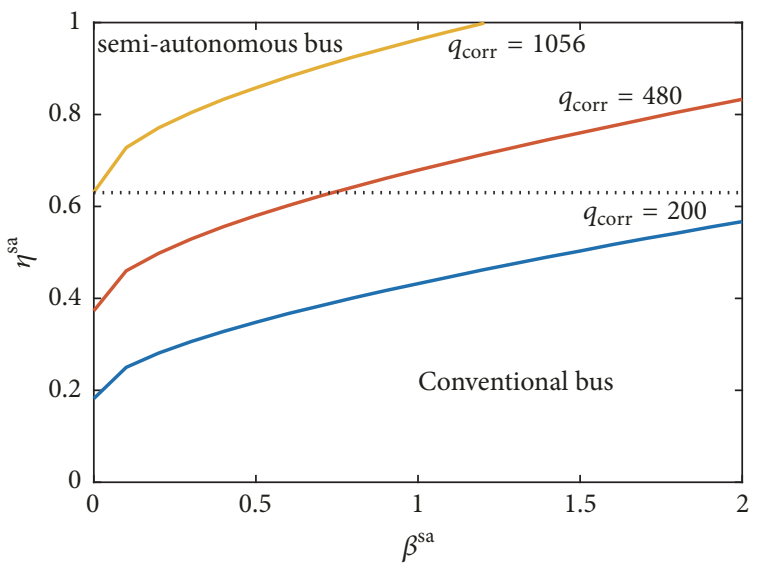

(b)

FIGURE 5: (a) Total cost and (b) applicability of semi-autonomous buses with respect to different levels of demand on the corridor. Note that the top-left side of each curve in (b) indicates the preferable region of semi-autonomous buses, whereas the other side is for conventional buses. The dotted line gives the upper bound of $\eta^{\text {sa }}$, which is 0.63 . Other parameters according to Table 2.

equivalent to conventional buses; no operating cost reduction is required, and thus the borderline crosses $(0,0)$. It can be seen that in the studied domain, the entire preferable region of fully autonomous buses is below the upper bound of $\eta^{\text {fa }}$, which means fully autonomous bus can be cost-efficient even if the capital investment is very high. By contrast, semi-autonomous buses are competitive only when $\beta^{\text {sa }} \leq$ 0.7 .

5.3. Impact of Demand Distribution. Section 4 shows that the choice between conventional buses and semi-autonomous buses depends on the ratio $q_{\text {wait }}^{\mathrm{c}} / q_{\text {wait }}^{\mathrm{sa}}$, representing how demand is distributed between the corridor and the branches, whereas the choice between conventional buses and fully autonomous buses is robust with respect to the demand distribution. Figure 5 shows the total cost as a function of the corridor demand with the other demand components held fixed. Also, the regions of applicability with respect to $\beta^{\text {sa }}$ and $\eta^{\text {sa }}$ of conventional buses and semi-autonomous buses, respectively, are shown considering three cases: the nominal $q_{\text {corr }}$, the lowest $q_{\text {corr }}$ satisfying the condition that the maximum load is on the corridor, and a high $q_{\text {corr }}$ (equal to 2.2 times the nominal demand). Generally, an increase in $q_{\text {corr }}$ means that waiting times on the corridor will have a larger influence on the total cost. It can be seen that the total cost for all types of buses rises as $q_{\text {corr }}$ increases. The performance of fully autonomous buses is superior compared to the other two types. 


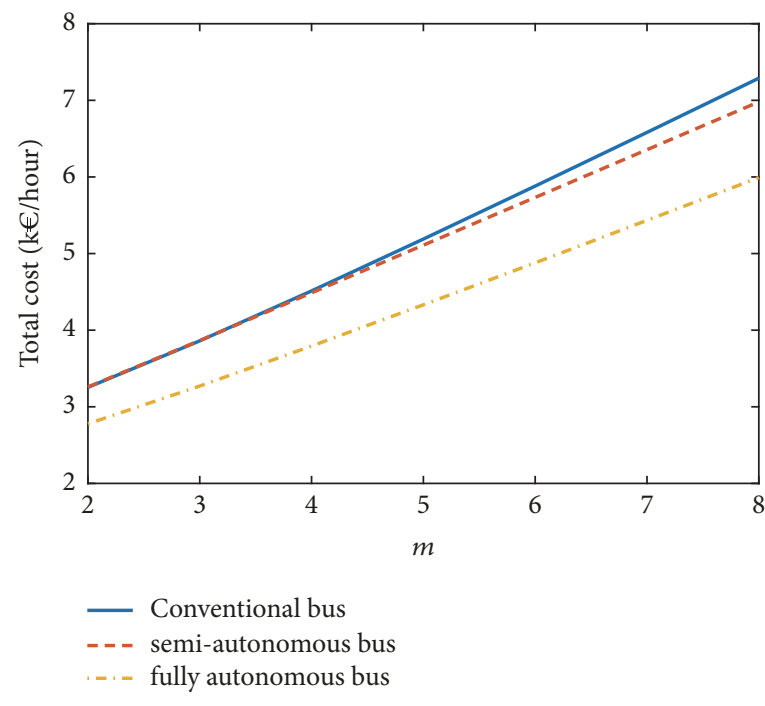

(a)

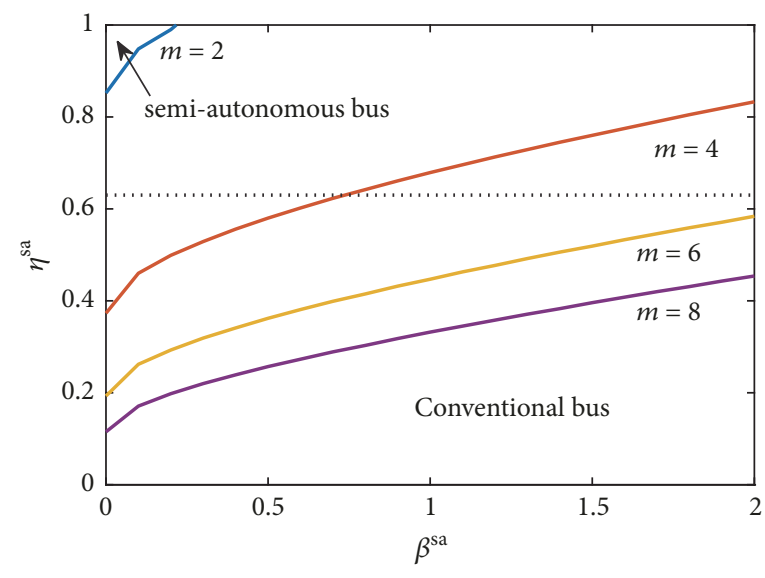

(b)

Figure 6: (a) Total cost and (b) applicability of semi-autonomous buses with respect to different $m$. Note that the top-left side of each curve in (b) indicates the preferable region of semi-autonomous buses, whereas the other side is for conventional buses. The dotted line gives the upper bound of $\eta^{\text {sa }}$, which is 0.63 . Other parameters according to Table 2.

The gap between conventional buses and semi-autonomous buses is not significant: the cross point in size appears when $q_{\text {corr }}=1021$ passengers/hour, and the total cost of semi-autonomous buses starts to surpass conventional buses when $q_{\text {corr }}=718$ passengers/hour. As $q_{\text {corr }}$ increases, semiautonomous buses tend to form shorter platoons. The platoon length approaches 1 at the highest corridor demand $q_{\text {corr }}$ (1056 passengers/hour), which means that the buses are driving separately. Figure 5(b) shows that as $q_{\text {corr }}$ increases, the preferred region of semi-autonomous buses shrinks, which means that semi-autonomous bus becomes less competitive. When $q_{\text {corr }}=1056$ passengers/hour, platooning does not yield any benefits even if semi-autonomous buses cost the same as conventional buses $\left(\beta^{\text {sa }}=0\right)$, because the labor saving is not enough to make up for the increase in users' cost. However, when $q_{\text {corr }}=m q_{\text {bran }}=200$ passengers/hour, semiautonomous buses can bring benefits even if the extra fixed capital cost is two times higher than conventional buses.

5.4. Impact of the Number of Branches. To study the impact of the network structure, the number of network branches $m$ is varied from 2 to 8 . Other parameters (e.g., $q_{\text {corr }}$ ) are held fixed, which means that the maximum load for each bus line $q_{\max }$ decreases as $m$ increases. More branches mean more bus lines, which should result in higher capital cost and operating cost. For semi-autonomous buses, meanwhile, larger $m$ also means more platooning opportunities.

Figure 6 demonstrates the impacts of $m$ on total cost and the applicability of semi-autonomous buses. With larger $m$, the bus size is reduced whereas the total cost increases for all types of buses. As in the previous sections, fully autonomous buses achieve the best performance among all vehicle types, so the following discussion focuses on the other two vehicle types. The semi-autonomous bus size is the most sensitive to the variation in $m$ among all bus types. However, the total savings (or losses) of semi-autonomous buses seem to be insignificant unless $m$ becomes extremely large (total savings yielding $309 € /$ hour when $m=8$ ). When there are only two branches, it is more cost-efficient to drive separately. Semi-autonomous buses start to gain advantage when $m=4$, below which conventional buses are preferable. As $m$ increases, semi-autonomous buses tend to form longer platoons, by seizing platooning opportunities so that the automation technology could take effect.

5.5. Impact of Corridor Length. The impact of network structure is further investigated by considering the driving time on the corridor $t_{\text {corr }}$ relative to the round-trip travel time $t_{\text {rnd }}$. When $t_{\text {rnd }}$ is fixed, the variation of $t_{\text {corr }}$ also results in different driving times on the branches, $t_{\mathrm{bran}}$. For all bus types, a rise in $t_{\text {corr }}$ means an increase in riding cost, according to (11). Although $t_{\text {bran }}$ goes down accordingly, the positive effect by a reduced riding cost on the branch can be easily canceled out by the increased riding cost on the corridor, given that $q_{\text {corr }}>m q_{\text {bran }}$. The ratio $\lambda=2 t_{\text {corr }} / t_{\text {rnd }}$ between corridor and total travel time also affects the performance of semiautonomous buses through the platooning rate, where a large $\lambda$ is expected to favor platooning.

Figure 7 shows the total cost and applicability of semiautonomous buses for different ratios between corridor and total travel time $\lambda$. When $\lambda \in[0,0.4]$, the semi-autonomous bus size is constant since all buses drive individually. Since semi-autonomous buses require more capital cost while the automation technology does not take effect due to the individual driving mode, semi-autonomous buses yield larger size and higher total cost than conventional buses. As $\lambda$ grows in the range $[0.4,1]$, semi-autonomous buses tend to form larger platoons. When $\lambda \in[0.6,1]$, semi-autonomous buses 


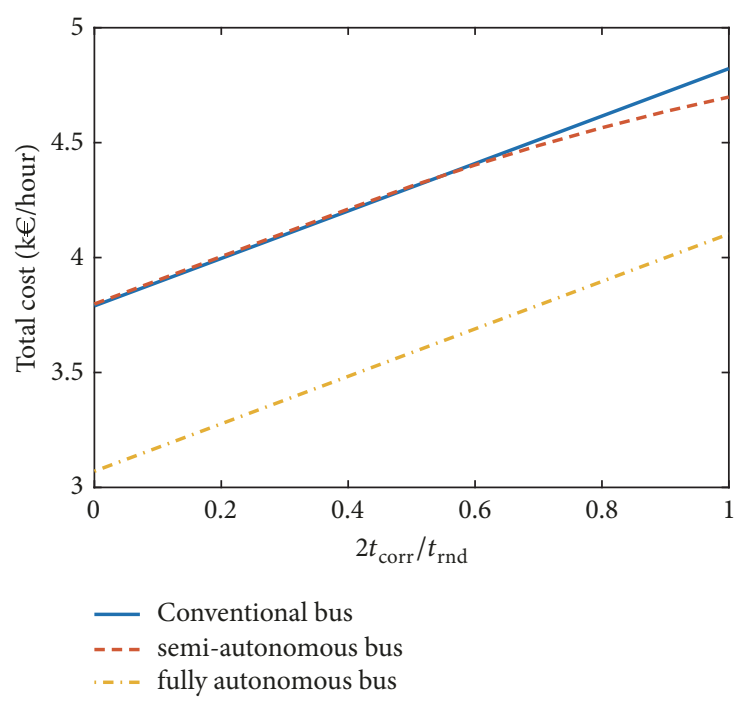

(a)

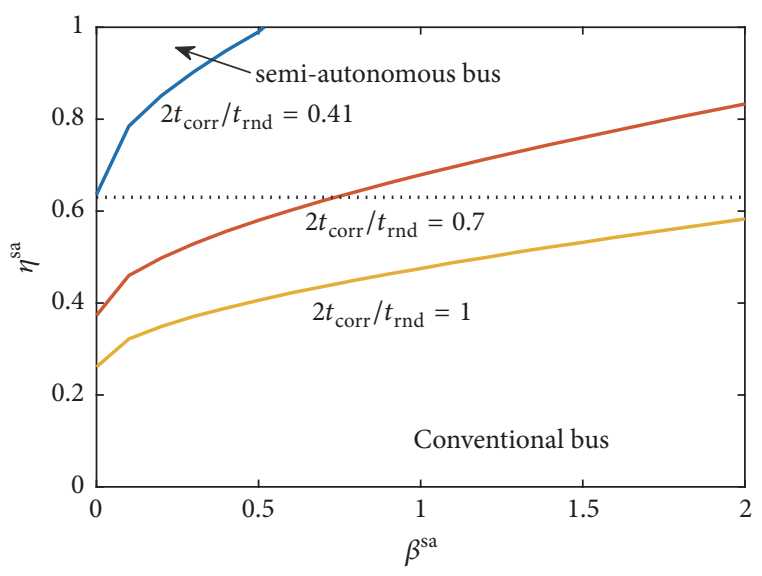

(b)

Figure 7: (a) Total cost and (b) applicability of semi-autonomous bus with respect to different ratios $\lambda$ between corridor and total travel time. Note that the top-left side of each curve in (b) indicates the preferable region of semi-autonomous buses, whereas the other side is for conventional buses. The dotted line gives the upper bound of $\eta^{\text {sa }}$, which is 0.63 . Other parameters according to Table 2.

become beneficial, as shown in Figure 7(a). In the extreme case when $\lambda=1$, the savings by semi-autonomous buses can be up to $124 € /$ hour. The applicability of semi-autonomous buses relies heavily on $\lambda$ and when this ratio is below 0.41 , semi-autonomous bus becomes inefficient even if no extra capital cost is incurred.

For the conventional and fully autonomous bus types, $\lambda$ only affects the riding cost, while other individual cost components and service characteristics remain unchanged as $\lambda$ varies. Since the riding costs are the same for these two bus types, the difference between the total costs is the same for any values of $\lambda$.

5.6. Impact of Autonomous Bus Speed. A sensitivity analysis is performed with respect to the speed ratios $\phi^{\text {sa }}$ and $\phi^{\text {fa }}$ in the domain [0.4 1.2], which is equivalent to the speed [6 18] km/hour assuming the nominal commercial speed 15 $\mathrm{km} /$ hour. Figure 8 shows the results on vehicle size, total cost, and the applicability of autonomous buses.

Semi-autonomous and fully autonomous buses exhibit the same pattern as the speed increases, with smaller vehicle sizes and lower total costs. However, fully autonomous buses are always more competitive at the same speed level. When it comes to applicability, semi-autonomous buses are more sensitive than fully autonomous buses to the speed level. Figure 8(c) shows that semi-autonomous buses lose their advantage completely when the speed is lowered by $1 \%$, whereas fully autonomous buses can still be beneficial even if the speed is lowered by $19 \%$. Assuming that the baseline commercial speed of conventional buses in urban areas is $15 \mathrm{~km} /$ hour, the minimum speeds required for semiautonomous bus and fully autonomous bus to be competitive are around $15 \mathrm{~km} /$ hour and $12 \mathrm{~km} /$ hour, respectively. If semi-autonomous buses can drive at $18 \mathrm{~km} /$ hour in urban areas, the total savings can be up to $614 € /$ hour.

\section{Conclusion}

The paper investigates the gains and losses associated with the operation of public transport services by conventional, semiautonomous, and fully autonomous buses. The generalized cost, composed of waiting, riding, operating, and capital cost, is modeled as a function of the bus size (directly and indirectly through headway and fleet size). The different vehicle types are assumed to differ in terms of fixed operating, capital costs, and, in some scenarios, commercial speed. A generic trunk-and-branches network is considered where the structure and demand profile are varied. Analytical optimal solutions are derived for all types of buses and the features of the three services, including bus size, headway and fleet size, as well as total cost, are compared. Furthermore, the losers and winners from implementing autonomous buses are identified. The following findings emerge from the analysis.

Fully autonomous buses exhibit great potential to benefit all stakeholders in the transport system, even if the additional capital expenditure is high. The reduction in crew cost, the main component of the current bus cost structure, compensates for the loss in capital cost and allows the operator to work with smaller buses and larger fleets. This reduces the service headway, which is an improvement of the level of service by shorter waiting times. However, based on current trials, the main handicap of this new technology is the commercial speed. The gains in the operating and waiting cost can be easily canceled out by the current speed level $6 \mathrm{~km} /$ hour, whereas the minimum commercial speed required for successful implementation is around $12 \mathrm{~km} /$ hour. Therefore, to extend the applicability of fully autonomous 

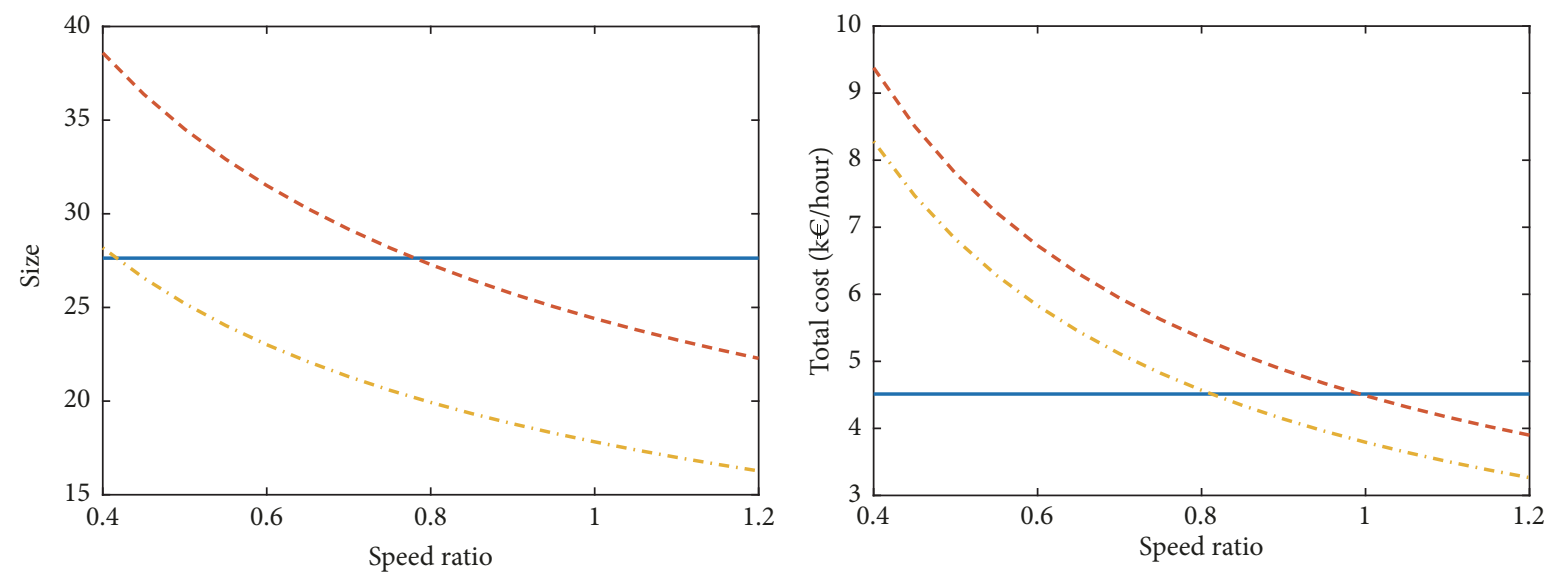

- Conventional bus

- - - $\phi^{\text {sa }}$ semi-autonomous bus

. - . - $\phi^{\mathrm{fa}}$ fully autonomous bus

(a)

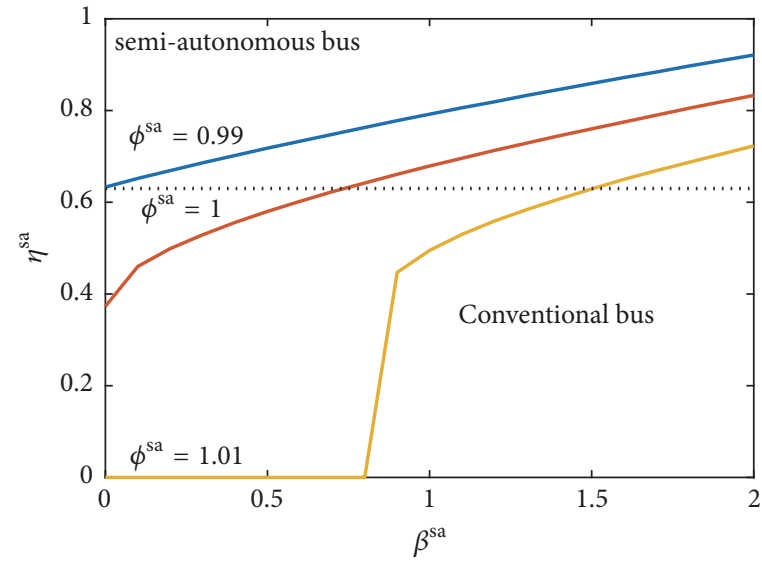

(c)
- Conventional bus

- - - $\phi^{\text {sa }}$ semi-autonomous bus

...- $\phi^{\mathrm{fa}}$ fully autonomous bus

(b)

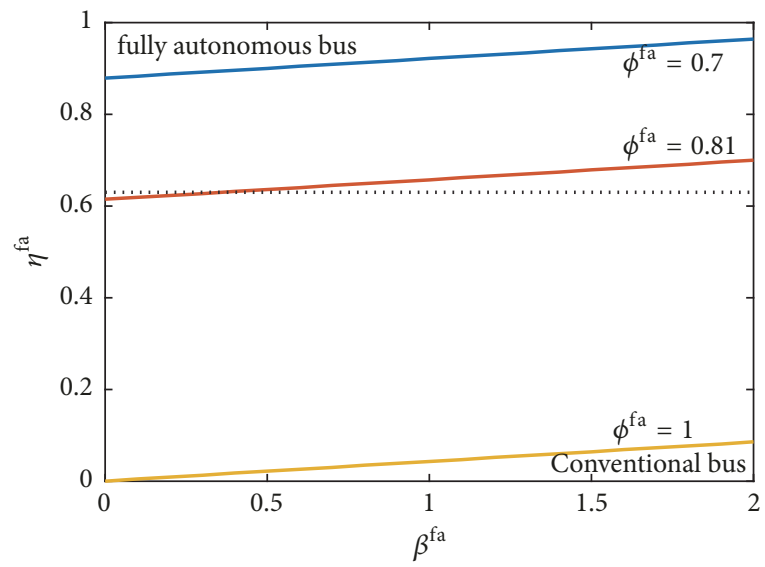

(d)

Figure 8: (a) Size, (b) total cost, (c) applicability of semi-autonomous buses, and (d) applicability of fully autonomous buses as functions of $\phi^{\text {sa }}$ and $\phi^{\text {fa }}$. Note that the top-left side of each curve in (c) and (d) indicates the preferable region of semi-autonomous (fully autonomous) bus, whereas the other side is for conventional bus. The dotted line gives the upper bound of $\eta^{\text {sa }}$, which is 0.63 . Other parameters according to Table 2 .

buses, the obstacle in the commercial speed needs to be overcome.

The advantages of semi-autonomous buses are more restricted. Although the platooning strategy enables reduction of operating cost by grouping buses together and removing drivers from the bus platoon followers, the side effect on the corridor users can be significant. The combined headways on the corridor are prolonged, leading to additional waiting cost. The numerical results show that the savings (if they exist) in the total cost are limited and conditional on many factors, including the relative length and demand distribution of the corridor and the branches. The service is beneficial mainly in scenarios with low demand along a long common corridor and high demand to and along the branches. The results suggest that services with platooning semi-autonomous vehicles are of most interest in interurban rather than intraurban settings.
One underlying cause of the modest benefits of semiautonomous buses in most scenarios is the insufficient platoon opportunities. To boost platooning, several measures could be taken, for example, replanning the existing bus transit network so that enough buses can be gathered on the common corridor. In this sense, platooning not only relies on the static network structure, but also triggers challenges in planning (e.g., scheduling and routing). It is also possible to promote the penetration rate of semi-autonomous commercial vehicles and enable $\mathrm{V} 2 \mathrm{~V}$ communication between buses and other vehicle types. Other challenges related to semiautonomous services that need further study include the time required to form the platoons and the management of manual drivers at the junction between the corridor and the branches.

This study analyzed different scenarios of demand where the bus mode does not have capacity problems. In this setting, the analysis suggests that autonomous vehicles may 
not be economically attractive to put in service until the technology of fully autonomous vehicles has matured sufficiently. However, outside of this range of values, where high capacity modes like tram or metro are more suitable, semiautonomous buses could be a good alternative since they allow the bus mode to gain capacity without an increase of drivers. An interesting direction for future research would be to find the gaps where semi-autonomous buses will improve the current transit systems.

\section{Appendix}

\section{A. Derivation of Maximum Load}

Assume that demand is uniformly distributed on the respective routes, which means the demand densities $\rho_{\text {corr }}, \rho_{\text {bran }}$, $\rho_{\text {full }}$ are all constants. For any two positions $y$ and $z$ on the corridor $\left(0 \leq y<z \leq l_{\text {corr }}\right.$, where $l_{\text {corr }}$ is the length of the corridor), the demand from $y$ to $z$ is $\rho_{\text {corr }}$ and the following relationship exists:

$$
\int_{0}^{l_{\text {corr }}} \int_{y}^{l_{\text {corr }}} \rho_{\text {corr }} \mathrm{d} z \mathrm{~d} y=q_{\text {corr }} .
$$

Therefore, the demand density for each bus line on the corridor is

$$
\frac{\rho_{\text {corr }}}{m}=\frac{2 q_{\text {corr }}}{m l_{\text {corr }}^{2}} .
$$

Similarly, the demand density for each bus line on the branch and the full route, respectively, is

$$
\begin{gathered}
\rho_{\text {bran }}=\frac{2 q_{\text {bran }}}{l_{\text {bran }}^{2}}, \\
\rho_{\text {full }}=\frac{q_{\text {full }}}{l_{\text {corr }} l_{\text {bran }}} .
\end{gathered}
$$

It is likely that demand is larger in the city center than in the suburbs, so we assume that the maximum load always appears on the corridor $\mathrm{AB}$. For any position $x$ on the corridor, $0 \leq x \leq l_{\text {corr }}$, the passenger load at $x$ for each bus line is

$$
\begin{gathered}
\int_{0}^{x} \int_{x}^{l_{\text {corr }}} \frac{\rho_{\text {corr }}}{m} \mathrm{~d} z \mathrm{~d} y+\int_{0}^{x} \int_{l_{\text {corr }}}^{l_{\text {corr }}+l_{\text {bran }}} \rho_{\text {full }} \mathrm{d} z \mathrm{~d} y \\
=-\frac{\rho_{\text {corr }}}{m} x^{2}+\left(\frac{\rho_{\text {corr }} l_{\text {corr }}}{m}+\rho_{\text {full }} l_{\text {bran }}\right) x .
\end{gathered}
$$

The first-order condition gives the optimal $x$, which is

$$
x^{*}=\frac{l_{\text {corr }}}{2}\left(1+\frac{m q_{\text {full }}}{2 q_{\text {corr }}}\right)
$$

by substituting (A.2) and (A.3) into (A.4). Thus, the maximum load is obtained:

$$
q_{\max }=\frac{\left(m q_{\text {full }}+2 q_{\text {corr }}\right)^{2}}{8 m q_{\text {corr }}} .
$$

Besides the maximum load on the corridor, it is also possible to calculate the maximum load on the branch. For any position $x$ on the corridor, $l_{\text {corr }} \leq x \leq l_{\text {corr }}+l_{\text {bran }}$, the load at $x$ for each bus line is

$$
\begin{aligned}
\int_{x}^{l_{\text {corr }}+l_{\text {bran }}} \int_{l_{\text {corr }}}^{x} \rho_{\text {bran }} \mathrm{d} y \mathrm{~d} z+\int_{x}^{l_{\text {corr }}+l_{\text {bran }}} \int_{0}^{l_{\text {corr }}} \rho_{\text {full }} \mathrm{d} y \mathrm{~d} z \\
=\rho_{\text {bran }}\left(x-l_{\text {corr }}\right)\left(l_{\text {corr }}+l_{\text {bran }}-x\right) \\
\quad+\rho_{\text {full }} l_{\text {corr }}\left(l_{\text {corr }}+l_{\text {bran }}-x\right) \\
=\rho_{\text {bran }}\left[-x^{2}+\left(2 l_{\text {corr }}+l_{\text {bran }}\right) x-l_{\text {corr }}^{2}-l_{\text {corr }} l_{\text {bran }}\right] \\
\quad+\rho_{\text {full }}\left(-l_{\text {corr }} x+l_{\text {corr }}^{2}+l_{\text {corr }} l_{\text {bran }}\right) .
\end{aligned}
$$

The first-order condition gives the $x$ at which load is at maximum:

$$
x^{*}=\frac{2 l_{\text {corr }}+l_{\text {bran }}}{2}-\frac{l_{\text {corr }} \rho_{\text {full }}}{2 \rho_{\text {bran }}} .
$$

The maximum load on the corridor $q_{\max }^{\text {bran }}$ is obtained by substituting (A.8) into (A.7):

$$
q_{\max }^{\text {bran }}=\frac{\left(q_{\text {full }}+2 q_{\text {bran }}\right)^{2}}{8 q_{\text {bran }}} .
$$

It can be easily noted that $q_{\max }^{\text {bran }}$ is analogous to $q_{\max }$ in (A.6) when $m=1$.

To satisfy the assumption that the maximum load along each bus line appears on the corridor rather than on the branch, the demands $q_{\text {corr }}, q_{\text {full }}$, and $q_{\text {bran }}$ must fulfill the following constraint:

$$
\frac{\left(m q_{\text {full }}+2 q_{\text {corr }}\right)^{2}}{m q_{\text {corr }}} \geq \frac{\left(q_{\text {full }}+2 q_{\text {bran }}\right)^{2}}{q_{\text {bran }}},
$$

where equality holds when $q_{\text {corr }}=m q_{\text {bran }}$.

\section{B. Derivation of Riding Times}

The speed is dependent on the bus type, whereas the total riding distance is calculated by

$$
\begin{aligned}
L_{\text {ride }}= & \int_{0}^{l_{\text {corr }}} \int_{0}^{z} \rho_{\text {corr }}(z-y) \mathrm{d} y \mathrm{~d} z \\
& +m \int_{l_{\text {corr }}}^{l_{\text {corr }}+l_{\text {bran }}} \int_{0}^{l_{\text {corr }}} \rho_{\text {full }}(z-y) \mathrm{d} y \mathrm{~d} z \\
& +m \int_{0}^{l_{\text {bran }}} \int_{0}^{z} \rho_{\text {bran }}(z-y) \mathrm{d} y \mathrm{~d} z \\
= & \frac{q_{\text {corr }} l_{\text {corr }}}{3}+\frac{m q_{\text {full }}\left(l_{\text {corr }}+l_{\text {bran }}\right)}{2} \\
& +\frac{m q_{\text {bran }} l_{\text {bran }}}{3} .
\end{aligned}
$$


TABLE 3: Original data used in the model. The size consists of seated and standing capacity. The overhead and profit margin are based on the total operating cost, which is the sum of the on-vehicle crew cost and the distance-related direct operating cost. Data source: Australian Transport Council [7].

\begin{tabular}{lcccccc}
\hline Type & Size (pax.) & $\begin{array}{c}\text { Annual capital } \\
\text { cost }\left(\times 10^{3} €\right)\end{array}$ & $\begin{array}{c}\text { On-vehicle crew costs } \\
(€ / \text { vehicle/hour })\end{array}$ & $\begin{array}{c}\text { Vehicle (direct operating) } \\
\text { costs }(€ / \text { bus } / \mathrm{km})\end{array}$ & Overhead (\%) & Profit margin (\%) \\
\hline Mini & 19 & 9.95 & 20.79 & 0.39 & 0.49 & 21 \\
Midi & 40 & 17.14 & 20.79 & 0.57 & 21 & 6 \\
Rigid standard & 64 & 23.31 & 20.79 & 0.62 & 21 & 6 \\
Rigid long & 81 & 24.51 & 20.79 & 0.71 & 21 & 6 \\
Articulated & 101 & 36.79 & 20.79 & & & 6 \\
\hline
\end{tabular}

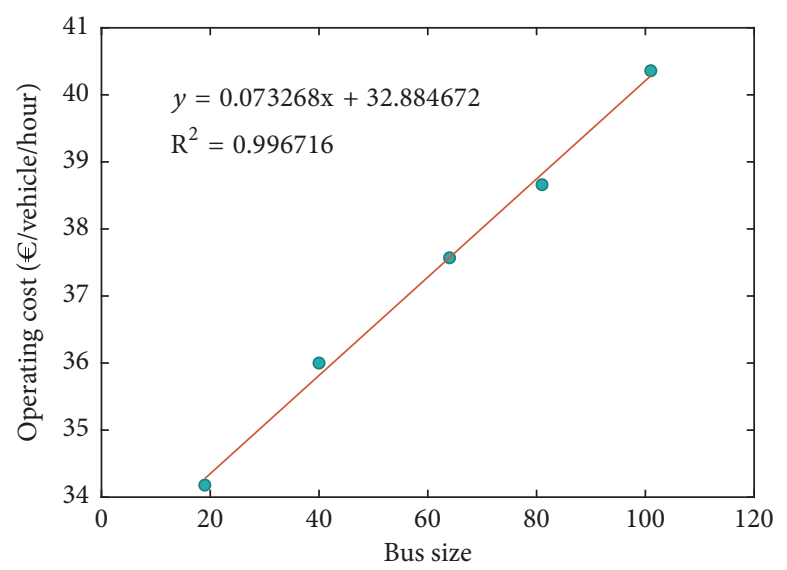

(a)

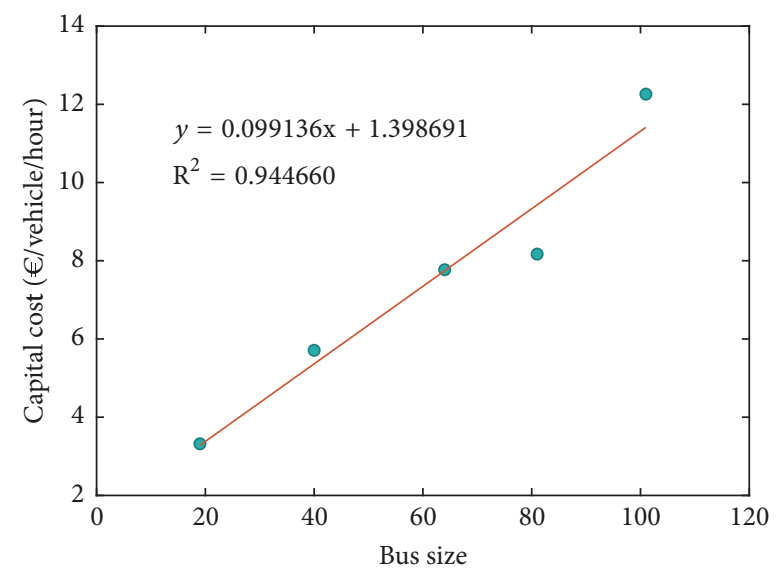

(b)

Figure 9: (a) Hourly operating cost per vehicle with respect to bus size; (b) hourly capital cost per vehicle with respect to bus size. Data source: Australian Transport Council [7].

Given the nominal speed $v_{\text {nom }}$, the round-trip riding cost is obtained:

$$
\begin{aligned}
& C_{\text {ride }}^{\mathrm{c}}=\frac{c_{\text {ride }}}{3 v_{\text {nom }}}\left[2 q_{\text {corr }} l_{\text {corr }}+3 m q_{\text {full }}\left(l_{\text {corr }}+l_{\text {bran }}\right)\right. \\
& \left.\quad+2 m q_{\text {bran }} l_{\text {bran }}\right] .
\end{aligned}
$$

Using the travel time $t_{\text {corr }}$ and $t_{\text {bran }}$, the riding cost can be written as

$$
\begin{aligned}
& C_{\text {ride }}^{\mathrm{c}}=\frac{c_{\text {ride }}}{3}\left[2 q_{\text {corr }} t_{\text {corr }}+3 m q_{\text {full }}\left(t_{\text {corr }}+t_{\text {bran }}\right)\right. \\
& \left.\quad+2 m q_{\text {bran }} t_{\text {bran }}\right] .
\end{aligned}
$$

\section{Derivation of Operating and Capital Cost Parameters}

The original data used to derive the operating and capital cost parameters are given in Table 3 . The linear regression result (based on Table 3) regarding the hourly operating and capital cost per vehicle with respect to the vehicle size is presented in Figure 9. Therefore, $c_{\text {oper }}=32.9 € /$ vehicle/hour, $b_{\text {oper }}=0.073$ $€ /$ vehicle/hour, $c_{\text {cptl }}=1.40 € /$ vehicle/hour, and $b_{\text {cptl }}=0.099$ $€ /$ vehicle/hour.

As mentioned in Section 3.3, the operating cost is the sum of on-vehicle crew cost (time-related), direct operating cost (distance-related), overhead, and profit margin. By dividing the annual capital cost with the equivalent hours per year (3000), the hourly capital cost can be obtained. The distancerelated cost can be converted to time-related cost, given the constant speed $15 \mathrm{~km} /$ hour. Taking the overhead and profit margin into account, the hourly operating cost is calculated. For example, the hourly operating cost for minibus is

$$
(20.79+0.39 \times 15) \times(1+21 \%) \times(1+6 \%)=34.17
$$

whereas the hourly capital cost for minibus is

$$
\frac{9.95 \times 1000}{3000}=3.32 \text {. }
$$

The linear regression model gives $c_{\text {oper }}=32.9 € /$ vehicle/hour, among which the on-vehicle costs are eliminated, which gives the nominal values of $\eta^{\text {sa }}$ and $\eta^{\mathrm{fa}}$ :

$$
\frac{20.79}{32.9}=0.63
$$

\section{Data Availability}

This paper contains analytical solutions and numerical results. All parameters needed are already given in the paper. 


\section{Conflicts of Interest}

The authors declare that there are no conflicts of interest regarding the publication of this article.

\section{Acknowledgments}

We thank Dr. Marcus Sundberg for the early discussions about the ideas. This work was supported by China Scholarship Council and TRENoP Strategic Research Area. The support is gratefully acknowledged.

\section{References}

[1] D. J. Fagnant and K. Kockelman, "Preparing a nation for autonomous vehicles: Opportunities, barriers and policy recommendations," Transportation Research Part A: Policy and Practice, vol. 77, pp. 167-181, 2015.

[2] D. J. Fagnant, K. M. Kockelman, and P. Bansal, "Operations of shared autonomous vehicle fleet for Austin, Texas, market," Transportation Research Record: Journal of the Transportation Research Board, vol. 2536, pp. 98-106, 2015.

[3] P. M. Bösch, F. Becker, H. Becker, and K. W. Axhausen, "Costbased analysis of autonomous mobility services," Transport Policy, 2017.

[4] A. Scheltes and G. H. d. A. Correia, "Exploring the use of automated vehicles as last mile connection of train trips through an agent-based simulation model: an application to Delft, Netherlands," International Journal of Transportation Science and Technology, vol. 6, no. 1, pp. 28-41, 2017.

[5] O. Bergqvist and M. Åstrand, Bus Line Optimisation Using Autonomous Minibuses [Bachelor's thesis], KTH Royal Institute of Technology, Stockholm, Sweden, 2017.

[6] S. Lam, A Methodology for The Optimization of Autonomous Public Transportation [Ph.D. thesis], University of New South Wales, Kensington, Australia, 2016.

[7] Australian Transport Council, "National guidelines for transport system management in Australia," 2006, https://atap.gov .au/technical-support-library/ngtsm/files/National_Guidelines_ Volume_4.pdf.

[8] S. M. Nourbakhsh and Y. Ouyang, "A structured flexible transit system for low demand areas," Transportation Research Part B: Methodological, vol. 46, no. 1, pp. 204-216, 2012.

[9] H. Badia, M. Estrada, and F. Robusté, "Bus network structure and mobility pattern: a monocentric analytical approach on a grid street layout," Transportation Research Part B: Methodological, vol. 93, pp. 37-56, 2016.

[10] W. Daniel and H. Martin, Automated Driving: Safer and More Efficient Future Driving, Springer, Cham, Switzerland, 2017.

[11] Antonio Loro Consulting Inc., "Planning for automated vehicles in Edmonton,” 2016, https://www.edmonton.ca/city_government/documents/RoadsTraffic/2016_automated_vehicles_report.pdf.

[12] TRB's E-Circular 232, "Automated vehicles symposium 2017: summary of a symposium," 2017, http://onlinepubs.trb.org/ onlinepubs/circulars/ec232.pdf.

[13] W. Zhang, E. Jenelius, and X. Ma, "Freight transport platoon coordination and departure time scheduling under travel time uncertainty," Transportation Research Part E: Logistics and Transportation Review, vol. 98, pp. 1-23, 2017.
[14] S. Torabi and M. Wahde, "Fuel-efficient driving strategies for heavy-duty vehicles: a platooning approach based on speed profile optimization," Journal of Advanced Transportation, vol. 2018, Article ID 4290763, 12 pages, 2018.

[15] T. Liu, A. Ceder, and A. Rau, "Scheduled platoons of public transport autonomous modular vehicles," 2018, https://www .events.tum.de/frontend/index.php?page id=3316.

[16] J. O. Jansson, "A simple bus line model for optimisation of service frequency and bus size," Journal of Transport Economics and Policy, pp. 53-80, 1980.

[17] P. White, Public Transport: Its Planning, Management and Operation, Taylor \& Francis, 5th edition edition, 2009.

[18] R. Oldfield and P. Bly, "An analytic investigation of optimal bus size," Transportation Research Part B: Methodological, vol. 22, no. 5, pp. 319-337, 1988.

[19] M.-C. Shih and H. S. Mahmassani, "Vehicle sizing model for bus transit networks," Transportation Research Record, no. 1452, pp. 35-41, 1994.

[20] S. Jara-Diaz and A. Gschwender, "Towards a general microeconomic model for the operation of public transport," Transport Reviews, vol. 23, no. 4, pp. 453-469, 2003.

[21] L. dell'Olio, A. Ibeas, and F. Ruisánchez, "Optimizing bus-size and headway in transit networks," Transportation, vol. 39, no. 2, pp. 449-464, 2012.

[22] T. Stephens, J. Gonder, Y. Chen, Z. Lin, C. Liu, and D. Gohlke, "Estimated bounds and important factors for fuel use and consumer costs of connected and automated vehicles," Tech. Rep. NREL/TP-5400-67216, National Renewable Energy Lab (NREL), Golden, CO, USA, 2016.

[23] A. Ceder, Public Transit Planning and Operation: Theory, Modeling and Practice, Elsevier, Butterworth-Heinemann, Oxford, UK, 2007.

[24] Z. Wadud, "Fully automated vehicles: a cost of ownership analysis to inform early adoption," Transportation Research Part A: Policy and Practice, vol. 101, pp. 163-176, 2017. 


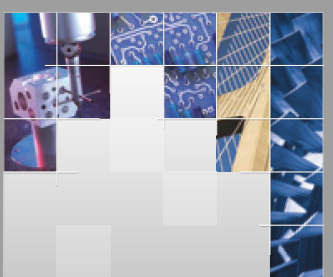

\section{Enfincering}
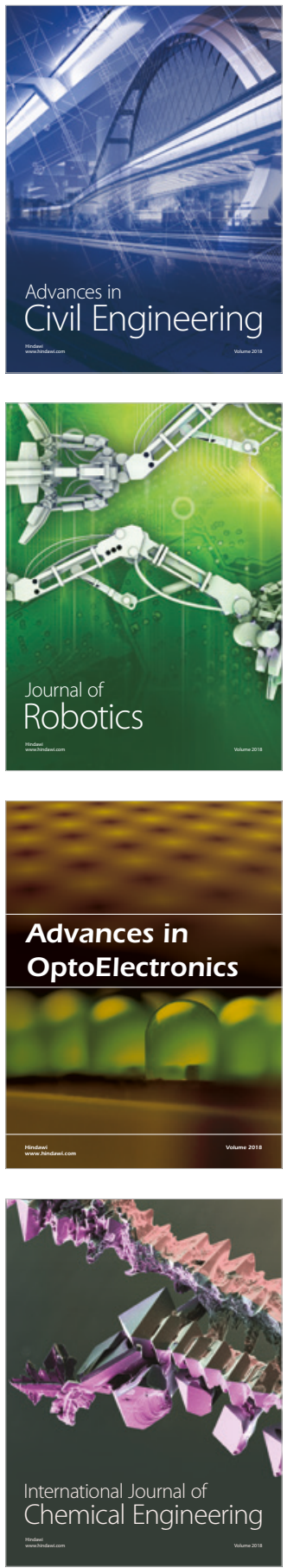

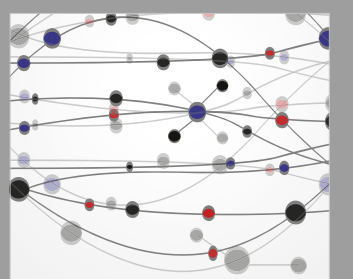

\section{Rotating \\ Machinery}

The Scientific World Journal

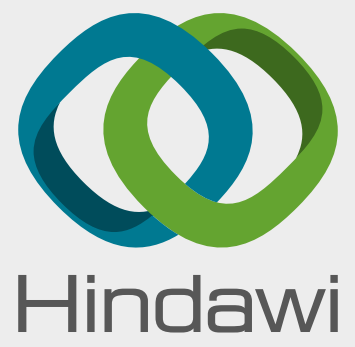

Submit your manuscripts at

www.hindawi.com
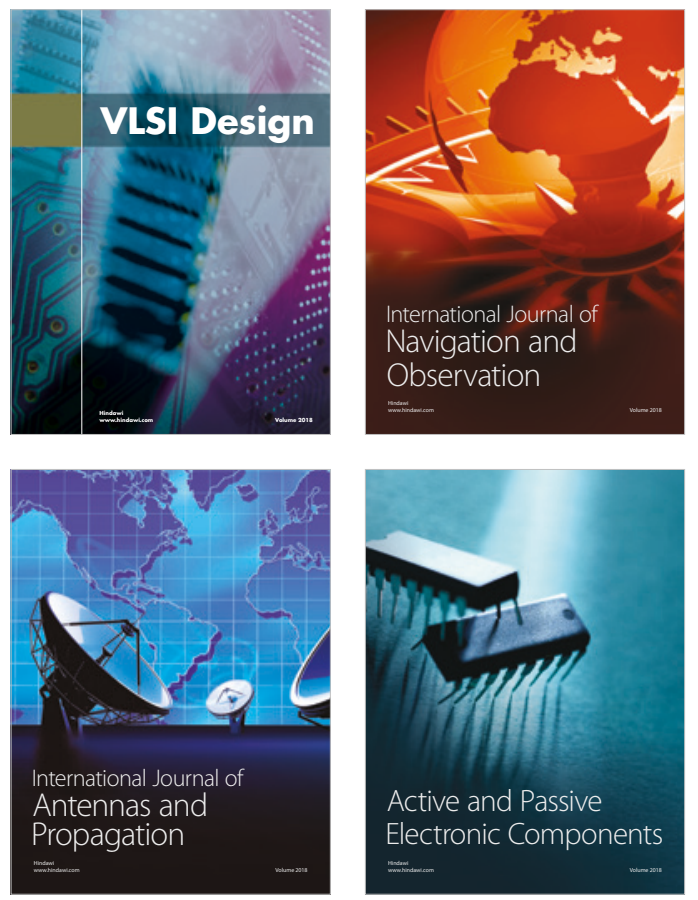
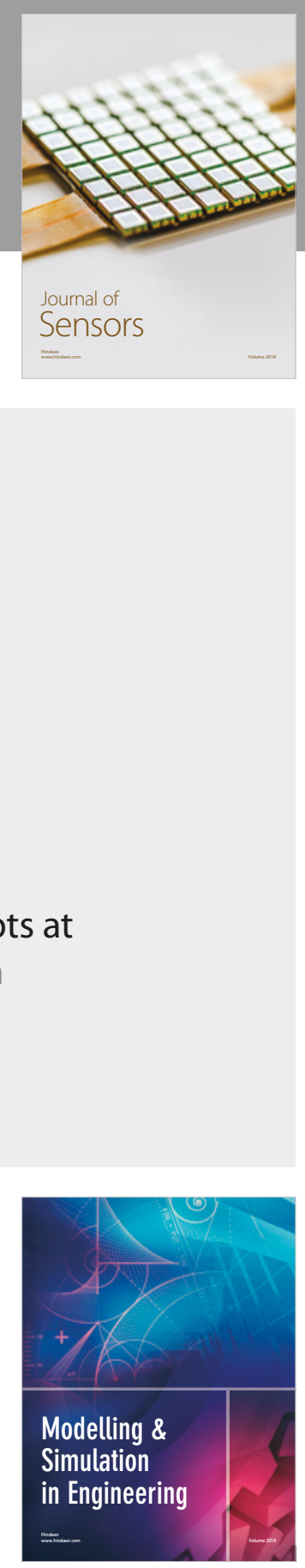

\section{Advances \\ Multimedia}
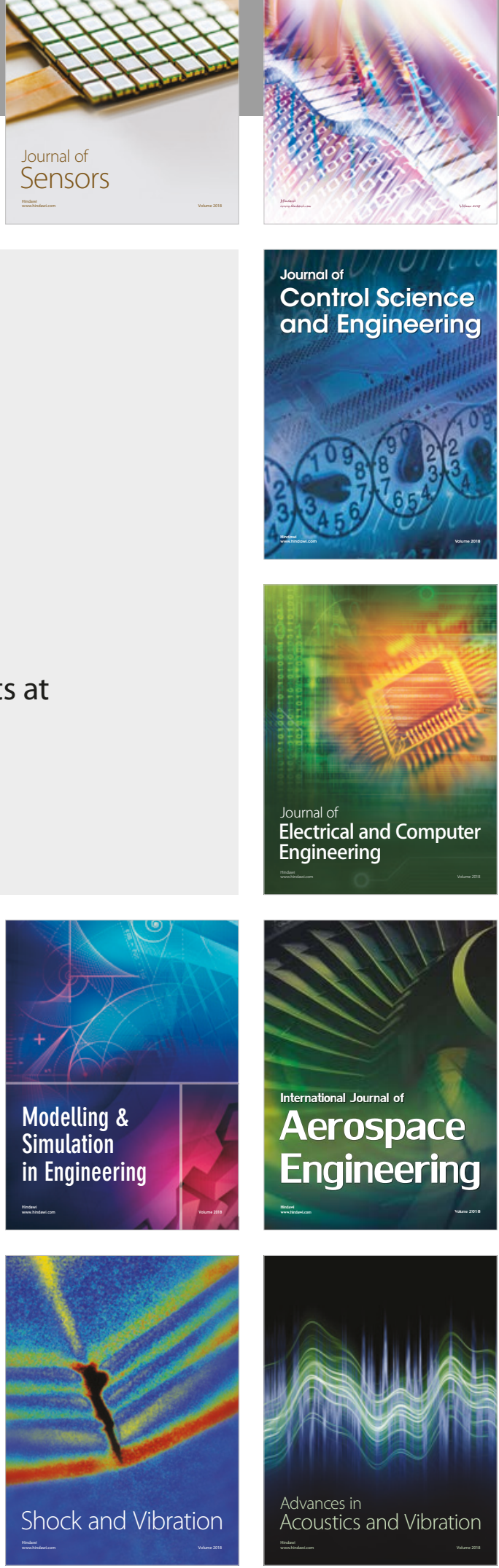